\title{
Immunotherapeutic effects of intratumoral nanoplexed poly I:C
}

\author{
M. Angela Aznar ${ }^{1 *+}$, Lourdes Planelles ${ }^{2 \dagger}$, Mercedes Perez-Olivares ${ }^{2}$, Carmen Molina $^{1}$, Saray Garasa', Iñaki Etxeberría , \\ Guiomar Perez ${ }^{1}$ Inmaculada Rodriguez ${ }^{1}$, Elixabet Bolaños ${ }^{1}$, Pedro Lopez-Casas², Maria E. Rodriguez-Ruiz ${ }^{1}$, \\ Jose L. Perez-Gracia ${ }^{4,5,6}$, Ivan Marquez-Rodas ${ }^{3}$, Alvaro Teijeira ${ }^{1,5,6}$, Marisol Quintero ${ }^{2+}$ and Ignacio Melero ${ }^{1,4,5,6^{*}+}$ (D)
}

\begin{abstract}
Poly I:C is a powerful immune adjuvant as a result of its agonist activities on TLR-3, MDA5 and RIG-I. BO-112 is a nanoplexed formulation of Poly I:C complexed with polyethylenimine that causes tumor cell apoptosis showing immunogenic cell death features and which upon intratumoral release results in more prominent tumor infiltration by T lymphocytes. Intratumoral treatment with BO-112 of subcutaneous tumors derived from MC38, 4T1 and B16F10 leads to remarkable local disease control dependent on type-1 interferon and gamma-interferon. Some degree of control of non-injected tumor lesions following BO-112 intratumoral treatment was found in mice bearing bilateral B16-OVA melanomas, an activity which was enhanced with co-treatment with systemic anti-CD137 and anti-PD-L1 mAbs. More abundant CD8 ${ }^{+}$T lymphocytes were found in B16-OVA tumor-draining lymph nodes and in the tumor microenvironment following intratumoral BO-112 treatment, with enhanced numbers of tumor antigen-specific cytotoxic T lymphocytes. Genome-wide transcriptome analyses of injected tumor lesions were consistent with a marked upregulation of the type-I interferon pathway. Inspired by these data, intratumorally delivered BO-112 is being tested in cancer patients (NCT02828098).
\end{abstract}

Keywords: BO-112, Intratumoral immunotherapy, Nanoplexed poly I:C

\section{Background}

Intratumoral local immunotherapy is gaining interest as a way to broaden the therapeutic window of immunotherapy agents and confine their effects to the tumor microenvironment and tumor-draining lymph nodes (TDLN) [1]. Moreover, a number of examples indicate that following intratumoral release, therapeutic effects against distant disease are observed beyond the injected tumor [1-3]. Immunotherapy agents in the form of cytokines $[4,5]$, recombinant viruses [6, 7], monoclonal antibodies (mAbs) [8], and pathogen-associated molecular patterns [9-11] can be delivered by intratumoral approaches.

Poly I:C is an analogue of double-stranded viral RNA that acts as an agonist of innate immune receptors deployed to detect infection by such microorganisms.

\footnotetext{
* Correspondence: maznargo@alumni.unav.es; imelero@unav.es

${ }^{\dagger} \mathrm{M}$. Angela Aznar and Lourdes Planelles contributed equally to this work.

${ }^{\dagger}$ Marisol Quintero and Ignacio Melero will share credit for senior authorship.

${ }^{1}$ Center for Applied Medical Research (CIMA), University of Navarra, Avenida

Pio XII, 55, 31008 Pamplona, Spain

Full list of author information is available at the end of the article
}

Endosomal TLR3 and intracellular MDA5 and RIG-I may detect the compound leading to upregulation of type-I interferon (IFN-I) and other proinflammatory pathways $[12,13]$. Indeed, Poly I:C was originally described for its effects as an exogenous IFN $\alpha / \beta$ inducer [14] with well documented antiviral and antitumor effects in mice $[15,16]$. These include immunotherapeutic efficacy observed following intratumoral injections [16-18] and it has been extensively used as a vaccine adjuvant including cancer vaccines [19-22].

A number of compounds have been produced to exploit the proimmune effects of Poly I:C in the clinic. Among these are Ampligene [23], Hiltonol [24-26] and BO-112 [27]. Hiltonol, a Poly I:C formulation stabilized by poly-L-lysine, is the most advanced of such compounds in the clinic, as it has been tested subcutaneously in healthy volunteers [28] and in cancer patients when given intratumorally $[10,29]$ and intramuscularly [30]. In healthy volunteers, subcutaneous injection induces prominent transient inflammation and a marked type-I interferon transcriptional signature among circulating 
PBMC [28]. BO-112 is a nanoplexed form of Poly I:C coupled to polyethylenimine (PEI) reminiscent of BO110 , a previous format of the compound that was found to in-vivo induce apoptosis in melanoma cells as a result of intense autophagy [31, 32].

In this article, we studied the immunotherapeutic profile of BO-112 following intratumoral delivery in experimental models. Our observations include induction of immunogenic cell death in a small fraction of tumor cells and objective immunotherapeutic activity dependent on Interferon- $\gamma$ (IFNY) and type-I interferon. Finally, we show the involvement of BATF-3-dependent conventional type-I dendritic cells $(\mathrm{cDC1})$ [33].

\section{Materials and methods}

\section{Animals and cell lines}

Animal studies were approved by the Ethics Committee of Animal Experimentation (CEEA) of the CNB and of the CIMA with compliance with national, institutional and EU guidelines. Six- to eight-week-old female BALB/c and $\mathrm{C} 57 \mathrm{Bl} / 6$ were purchased from Envigo (www.envigo. com). C57Bl/6 Batf $3^{\text {tm } 1 \mathrm{Kmm} / J}$ (Batf3 ${ }^{-1-}$ ) [33] and IFN-a/bR ${ }^{o / o}$ (IFNARKO) [34] were kindly provided, by Dr. Kenneth M. Murphy, Washington University, St. Louis, MO and by Matthew Albert (Institut Pasteur, Paris) respectively, and were bred at CIMA in specific pathogen-free conditions. Mice were housed in the Animal Facility of Centro Nacional de Biotecnologia (CNB-CSIC, Madrid, Spain) and Centro de Investigacion Medica Aplicada (CIMA, Pamplona, Spain).

B16-F10 mouse melanoma cells and 4 T1 mouse breast carcinoma were purchased from the ATCC, and B16-OVA melanoma cells and MC38 colon carcinoma cells were a kind gift from Dr. Lieping Chen (Yale University, New Heaven, CT) and Dr. Karl E. Hellström (University of Washington, Seattle, WA) respectively. Tumor cells were cultured in RPMI 1640 (Gibco) containing 10\% fetal bovine serum (FBS, Sigma-Aldrich), $2 \mathrm{mM}$ glutamine (Gln, Gibco), $100 \mathrm{U} / \mathrm{ml}$ penicillin and $100 \mu \mathrm{g} / \mathrm{ml}$ streptomycin $(100 \mathrm{U} / \mathrm{ml})$, and $50 \mu \mathrm{M}$ 2-mercaptoethanol (Gibco). The B16-OVA cell line was supplemented with $400 \mu \mathrm{g} / \mathrm{mL}$ Geneticin (Gibco). Cell lines were routinely tested for mycoplasma contamination (MycoAlert Mycoplasma Detection Kit, Lonza).

$\mathrm{UMBY}$ and ICNI human melanoma were derived from primary surgical samples of metastatic lesions of patients at the Department of Dermatology, University Hospital Erlangen and grown in DMEM (Gibco) containing 10\% FBS, $4 \mathrm{mM}$ Gln and 1\% P/S. HT-29 and HCT 116 colon cancer from the ATCC were cultured in RPMI, $2 \mathrm{mM}$ Gln, 10\% FBS and 1\% P/S. SK-BR-3 and BT-474 breast cancer cell lines were a kind gift from Dr. López-Botet, IMIM, Barcelona and were grown in DMEM/F12 (1:1) (Invitrogen), containing $2.5 \mathrm{mM}$ Gln, 10\% STF and $1 \% \mathrm{P} / \mathrm{S}$.
BO-112

BO-112 was developed and provided by Bioncotech Therapeutics (Madrid, Spain). All experiments were performed with the same batch.

\section{In vitro experiments}

The in vitro cytotoxicity of BO-112 in mouse and human cell lines was continuously assessed by measuring electric impedance in an xCELLigence machine (ACEA). Tumor cells $\left(1.5-2 \times 10^{5}\right)$ were seeded on specific 8-well plates to measure electric impedance. After 4-5 h, BO-112 or Poly I:C (Sigma) was added to culture media at identical concentrations in a final volume of $200 \mu \mathrm{L}$ per well. PEI (Polyplus-transfection ${ }^{\oplus}$ ) was added to culture media at the same concentrations as it is present in BO-112 formulation. Electric impedance was measured every five minutes for $48 \mathrm{~h}$. In vitro BO-112 cytotoxicity was also assessed by the CellTiter AQueous One Solution Cell Proliferation Assay (MTS, Promega). Briefly, tumor cells $\left(5 \times 10^{3}\right.$ cells/well; 96 flat-well plates, 8 replicates per condition) were cultured for $48 \mathrm{~h}$, alone or with BO-112 $(0.25,0.5$ and $1 \mu \mathrm{g} / \mathrm{mL})$ and absorbance (OD $492 \mathrm{~nm}$ ) measured in an ELISA plate reader. Three independent MTS assays were performed. Cell viability is referred to untreated cells (100\%).

For RNA expression analyses, B16-OVA cell lines were cultured $24 \mathrm{~h}$ with $\mathrm{BO}-112$ at $0.5 \mu \mathrm{g} / \mathrm{mL}$ or in the presence of equivalent volumes of BO-112 vehicle.

HMGB1 detection in culture supernatants was performed with HMGB1 ELISA detection kit following the manufacturer's instructions (IBL International ST51011).

\section{In vivo experiments}

B16-F10 and B16-OVA melanoma, MC38 colon carcinoma or $4 \mathrm{~T} 1$ breast carcinoma cells were injected subcutaneously $\left(5 \times 10^{5}-10^{6}\right)$ into the right flank of 8 - to 10-week-old female C57BL/6 or BALB/c (6-11 mice/ group) on day 0 . Tumors were measured twice per week with calipers and the volume calculated (length $\mathrm{x}$ width ${ }^{2} / 2$ ). When tumors reached a volume of $80-100 \mathrm{~mm}^{3}$ (day 0) mice were randomized into different groups of treatment according to the experiment. Poly I:C or BO-112 formulation $(2.5 \mathrm{mg} / \mathrm{Kg}, 100 \mu \mathrm{l})$, was administered by intratumoral injection twice per week for three weeks (six doses in total). The control group received intratumoral injections of $5 \%$ glucose (BO-112 vehicle, identical volume) or PEI (identical amount per dose as present in each dose of BO-112). Survival was monitored daily, and tumors were measured twice per week until the animals died or the tumor volume reached the maximum allowed size.

To evaluate the systemic antitumor effects, $5 \times 10^{5}$ (injected/treated tumor) and $1.5 \times 10^{5}$ (contralateral tumor) B16-OVA cells were injected into each flank respectively. For evaluation of $\mathrm{cDC} 1$ in $\mathrm{BO}-112$-meditated 
antitumor response, identical experiments were performed in $\mathrm{Batf}^{-1-}$ mice (in parallel with WT mouse groups). For evaluation of intratumoral BO-112 in combination with systemic immunostimulatory monoclonal antibodies, mice were intratumorally injected with $\mathrm{BO}-112$ or vehicle. The intratumoral treatment schedule was the same as that described for single tumor models. Starting at the second BO-112 administration, mice received concomitant intraperitoneal administration $(150 \mu \mathrm{g} / \mathrm{dose})$ of either InVivoPlus anti-PD-L1 (10F.9G2), InVivo anti-CD137 (3H3) or InVivoMAb rat IgG from BioXCell.

For flow cytometry, IHC and RNA extraction experiments, mice received two intratumoral administrations and were euthanized $48 \mathrm{~h}$ post-second dose.

\section{Flow cytometry}

Fresh tumors were excised from mice, weighed and mechanically dissected and enzymatically digested for $15-30 \mathrm{~min}$ at $37^{\circ} \mathrm{C}$ with appropriate medium: DMEM F-12 (Gibco), $1 \mathrm{mg} / \mathrm{mL}$ Collagenase 1A (Roche), $2.5 \mathrm{U} / \mathrm{mL}$ Dispase (Roche), $20 \mathrm{U} / \mathrm{mL}$ DNAse-I (Roche), $20 \mathrm{mM}$ HEPES (Lonza) and antibiotics. The enzymatic reaction was stopped with 5\% FBS in phosphate-buffered saline (PBS). After hypotonic lysis, cell aggregates were removed by filtering the cell suspension with a $70-\mu \mathrm{m}$ cell strainer (BD Falcon, BD Bioscience) and counted. Lymph nodes were excised from mice and mechanically disrupted and passed through a 70- $\mu \mathrm{m}$ cell strainer. Perfect count microspheres (Cytognos) were used as an internal standard according to the manufacturer's instructions to calculate absolute cell counts in cell suspensions.

Single cell suspensions from tumors and lymph nodes were previously treated with FcR-Block (anti-CD16/32 clone 2.4G2; BD Biosciences Pharmingen) and were then surface stained at $4{ }^{\circ} \mathrm{C}$ with fluorochrome-labeled antibody cocktails defined for each staining. Tetramer staining was performed according to the manufacturer's protocol. Flow cytometry antibodies, tetramers, cell death stainings and isotype controls are listed in Additional file 1. Table S1. For intracellular FOXP3 staining, cells were fixed and permeabilized using the True-Nuclear ${ }^{\mathrm{rm}}$ Transcription Factor Buffer Set (Biolegend).

Samples were acquired in a Gallios Cytometer (Beckman Coulter), a FACSCanto II (BD Biosciences) and a CytoFLEX Flow cytometer (BD Biosciences). Kaluza Flow Analysis Software (Beckman Coulter) and FlowJo (Treestar) software were used for data analysis.

\section{Depletion experiments}

100-300 $\mu \mathrm{g} /$ dose of anti-CD4 (GK1.5), anti-CD8 (2.43), anti-NK1.1 (PK136), anti-Gr1 (RB6-8C5) mAbs or Rat IgG2b (LTF-2) from BioXCell, were injected one day before therapy, concurrently with the first intratumoral injection and at days 3,7 , and 10 after the beginning of therapy. Cell depletion was validated in blood samples by flow cytometry analysis, showing a specific reduction of more than $95 \%$ of each respective cell subset. Gr1 depletion was confirmed in the tumor microenvironment on day 7 (the day of the first BO-112 injection). For IFN $\gamma$ neutralization, mice were treated with $250 \mu \mathrm{g}$ of anti-IFN $\gamma$ (XMG1.2) or rat IgG the day prior to each BO-112 treatment. Then, mice were injected weekly with for depletion maintenance $(100 \mu \mathrm{g} / \mathrm{dose})$.

\section{Tissue histology and immunostaining}

Formaldehyde-fixed and paraffin-embedded tissue sections $(3 \mu \mathrm{m}$ thick) were cut, dewaxed and hydrated. Heat induced antigen retrieval was applied for $30 \mathrm{~min}$ at $95^{\circ} \mathrm{C}$ in 0,01 M Tris-1 mM EDTA buffer $(\mathrm{pH}=9)$ in a Pascal pressure chamber (S2800, Dako). Sections were incubated overnight at $4{ }^{\circ} \mathrm{C}$ with anti-CD4 (1:1000; Abcam, ab183685) or anti-CD8 (1:300; Cell Signaling, 98,941) Visualisation was performed using MACH 2 rabbit AP-polymer (Biocare Medical, RALP525) with StayRed (Abcam, ab103741) as chromogen according to the manufacturer's instructions.

\section{RNA extraction}

Total RNA was isolated in two steps using TRIzol (Life technologies) and Rneasy Mini-Kit (Quiagen) purification, following the manufacturer's RNA cleanup protocol. The assessment of RNA integrity was performed with the Agilent 2100 bioanalyzer (Agilent Technologies) and high-quality RNA was hybridized to Affymetrix Clariom S Mouse Affymetrix microarrays following the manufacturer's protocol.

\section{Gene expression analysis}

The transcriptome experiment with Clariom S Mouse Affymetrix microarrays was normalized using the Robust Multichip Average (RMA) algorithm [35]. After quality assessment, a filtering process was performed to eliminate low expression probe sets. Applying the criterion of an expression value greater than 4 in at least 3 samples of one of the experimental conditions (BO-112 or control samples), 21,731 probe sets were selected for statistical analysis in the in vivo experiment. Regarding the differential expression analysis upon BO-112 incubation of B16-OVA in vitro, 18,412 probe sets were selected for statistical analysis after applying the criterion of an expression value greater than 4 in at least 2 samples of one of the experimental conditions (BO-112 or control samples).

Linear Models for Microarray Data (LIMMA) [36] was used to identify the probe sets that showed significantly differential expression between experimental conditions. Genes were selected as significant using a B-statistic cut-off $(\mathrm{B}>0)$. $\mathrm{R}$ and Bioconductor were used for preprocessing and statistical analysis [37]. 
The functional enrichment analysis was performed using Ingenuity Pathway Analysis (Ingenuity Systems, www.ingenuity.com), whose database includes manually curated and fully traceable data derived from literature sources. In addition, enrichment analyses of some gene sets of interest extracted from different publications [38, 39] were performed using the hypergeometric distribution in $\mathrm{R}$ [37]. Microarray expression data can be downloaded from Gene Expression Omnibus (GEO) under the Series accession number GSE116078.

\section{Statistical analysis}

Statistical analyses were performed using Prism software (GraphPad Software, Inc.). A two-tailed Student's t-test or Mann-Whitney tests were used to analyze statistical differences between groups. The Mantel-Cox test was used for survival analysis. For tumor growth data analyses, mean volumes of tumors over time were fitted using the formula $\mathrm{y}=\mathrm{A} \times \mathrm{e}(\mathrm{t}-\mathrm{t} 0) /(1+\mathrm{e}(\mathrm{t}-\mathrm{t} 0) / \mathrm{B})$, where $\mathrm{t}$ represents time, $\mathrm{A}$ the maximum size reached by the tumor and B its growth rate. Treatments were compared using the extra sum-of-squares $\mathrm{F}$ test. Values of $p<0.05$ ${ }^{(*)}, p<0.01^{(* *)}$ and $p<0.001^{(* * * *)}$ were considered significant.

\section{Results}

Intratumoral BO-112 controls transplanted syngeneic tumors and induces cell death in a fraction of malignant cells

BO-112 is a GMP-grade pharmaceutical composition of nanoplexed Poly I:C (300-5000 mer) coupled to polyethylenimine characterized by their monomodal diameter distribution (at least $90 \%$ of particles mono-modal diameter distribution bellow $300 \mathrm{~nm}$ ) and z-average diameter (less or equal $150 \mathrm{~nm}$ ) (PCTEP2016078078, Additional file 2: Figure S1). Previous forms of Poly IC-PEI nanocomplexes termed BO-110 have been intravenously delivered in mouse models giving rise to therapeutic effects against subcutaneous transplanted melanomas in a fashion related to its ability to induce tumor cell apoptosis in the context of intense autophagy elicited via MDA5 stimulation in malignant cells [31, 32].

In keeping with those findings, Fig. 1a shows that BO-112 induces death in a dose-dependent manner in cultured cell lines able to engraft in syngeneic mice representing melanoma (B16-F10, B16-OVA), colon cancer (MC38) and triple negative breast cancer (4 T1). Tumors derived from such cell lines are described as poorly immunogenic and difficult to treat with immunotherapy [40]. BO-112-induced cytotoxicity was also observed in a panel of human cell lines (Additional file 3: Figure S2A), including melanoma, colon cancer and breast cancer. Of particular importance, identical concentrations of Poly I:C were not cytotoxic in either mouse or human tumor cell lines (Fig. 1a and Additional file 3: Figure S2A). These results were further confirmed by measuring cytotoxicity in MTS assays in mouse and human cell lines (Additional file 3: Figure S2B).

To study the effects of intratumoral injection in subcutaneous malignant nodules derived from these tumor-cell lines upon engraftment in syngeneic mice, BO-112, Poly I:C or vehicle were repeatedly delivered intratumorally when tumors reached a volume of $80-100 \mathrm{~mm}^{3}$ (Fig. 1b and c). Figure $1 \mathrm{~b}$ shows the very clear therapeutic effects of BO-112 at halting and delaying B16-F10 tumor progression, that were not seen in the mice randomized to receive injection of either Poly I:C or vehicle. Representative photographs showing B16-OVA tumors at day 15 are shown in Fig. 1b (inset). BO-112 antitumor therapeutic effects were also observed in MC38- and 4T1-tumor bearing mice upon a similar repeated intratumoral treatment regimen with BO-112 (Fig. 1c).

Next, we examined whether BO-112 could induce local tumor cell death in an immunogenic fashion [41, 42]. In B16-OVA cultures, tumor cell death was abundant 24 and $48 \mathrm{~h}$ after exposure to $\mathrm{BO}-112$ in the form of apoptosis characterized by Annexin $\mathrm{V}$ binding and loss of plasma membrane integrity (7AAD staining), as shown in Fig. 2a. This increase in apoptotic cell subsets was also observed in BO-112-treated human cell lines (Additional file 3: Figure S2C). Interestingly, dying or dead cells showed molecular features associated with immunogenic cell death [42] including calreticulin (CRT) exposure on the outer leaf of the plasma membrane, and HMGB1 release to the culture supernatant and CD95 surface expression (Fig. 2b). Such cells also showed enhanced surface expression of MHC class I. Importantly, identical concentrations of Poly I:C added to the culture media failed to induce these hallmarks of immunogenic cell death (Fig. 2b) in tumor cells.

When treating subcutaneous tumors derived from B16-OVA (Fig. 2c), a fraction of apoptotic cells could be observed five days after the onset of intratumoral treatment. Interestingly, in CD45- cells obtained from the tumor nodules there was an increased surface expression of MHC-I, CRT and CD95 (Fig. 2c), suggesting features of immunogenic cell death in vivo. Of note, PEI itself (without Poly I:C) was not cytoxic in culture and did not affect progression of B16-OVA melanomas following repeated intratumoral administration in the same quantities as those present in BO112 (Additional file 4: Figure S3 A-C).

\section{Intratumoral administration is required for antitumor activity as opposed to subcutaneous delivery}

Since direct intratumoral injection of BO-112 therapeutically controlled tumor progression, we tested whether similar results could be achieved by subcutaneous delivery 
A
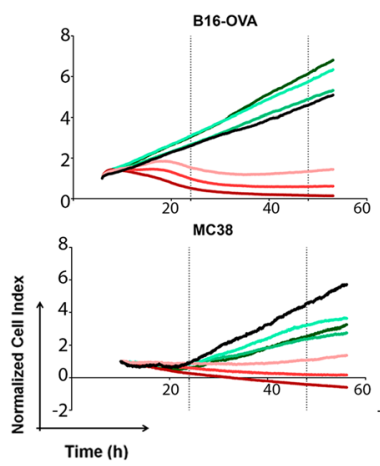

B

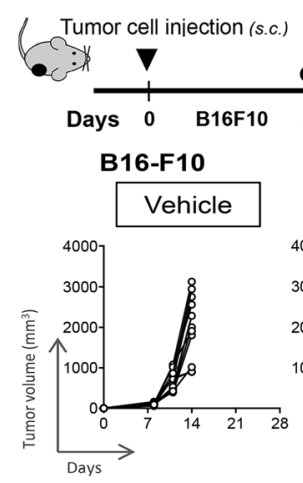

(? BO-112 or Poly I:C (i.t. $2.5 \mathrm{mg} / \mathrm{Kg}$ )
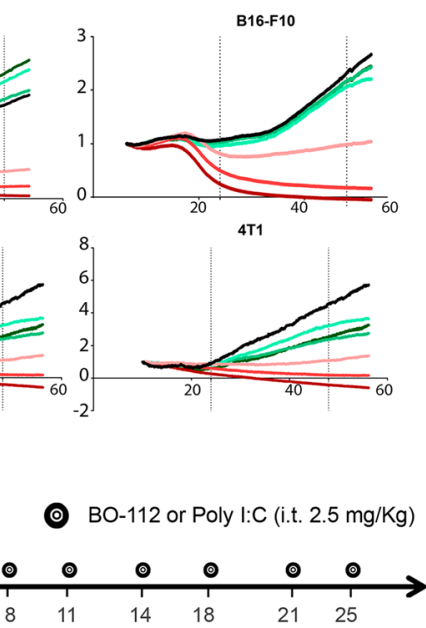

- Control

- $0,25 \mu \mathrm{g} / \mathrm{mL}$

- $0,5 \mu \mathrm{g} / \mathrm{mL}$

- $1 \mu \mathrm{g} / \mathrm{mL}$

- $0,25 \mu \mathrm{g} / \mathrm{mL}$

- $0,5 \mu \mathrm{g} / \mathrm{mL}]$ Poly I:C

- $1 \mu \mathrm{g} / \mathrm{mL}$
BO-112

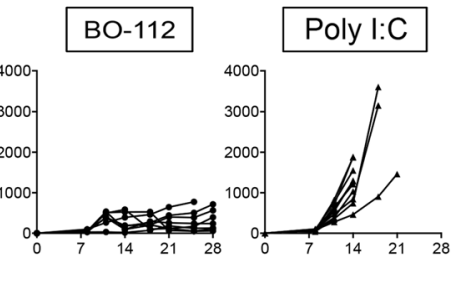

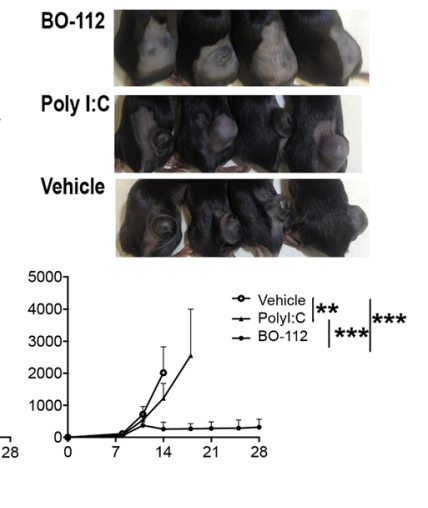

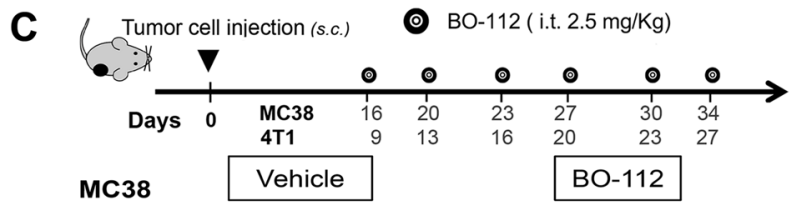
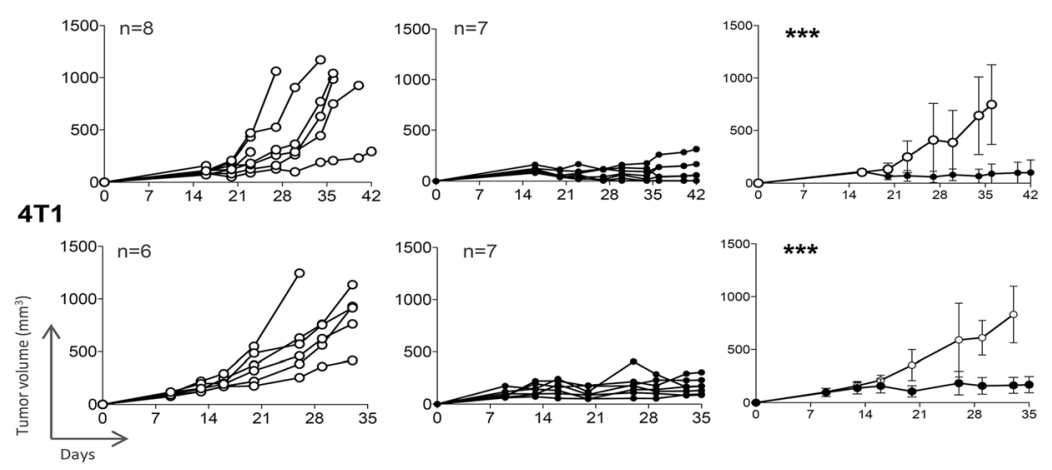

Fig. 1 Local injection of BO-112 exerts antitumor effects. a. Cell viability (in terms of electric impedance) of cultured tumor cell lines was measured in XCELLigence plates over time in the presence of different concentrations of BO-112 or Poly l:C as indicated, to study effects on cell viability. b. Tumor volume follow-up of in vivo engrafted syngeneic B16F10 tumors treated intratumorally with control vehicle, Poly I:C or BO-112 as indicated in the diagram. Representative photographs of mice treated with BO-112, Poly I:C or control vehicle are included as an inset. C. Individual follow-up of tumor volume means \pm SD (in graphs on the right) of MC38 and 4T1-bearing mice treated with BO-112 or control vehicle as indicated. Experiments are representative of two similarly performed. ${ }^{* *} P<0.001$

elsewhere in the mouse. Such experiments comparing intratumoral versus subcutaneous delivery at the same doses were performed in MC38 and B16-OVA tumor-bearing mice (Additional file 5: Figure S4). As shown in Additional file 5: Figure S4, while intratumoral delivery controlled tumor progression, this was not the case for subcutaneous administrations which failed to control tumor progression. Accordingly, intratumoral release is more efficacious than subcutaneous release under identical experimental conditions. 
A

Viability $24 \mathrm{~h}$ and $48 \mathrm{~h}$

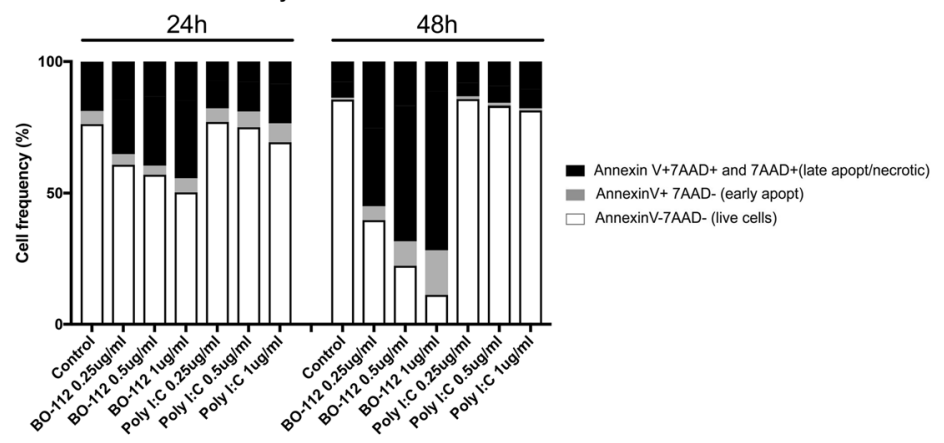

B
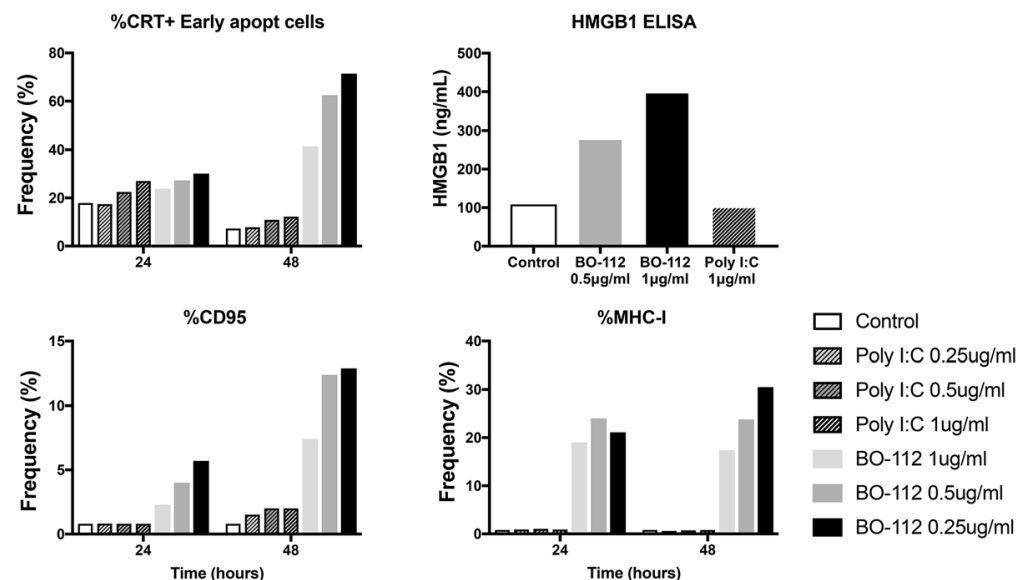

C
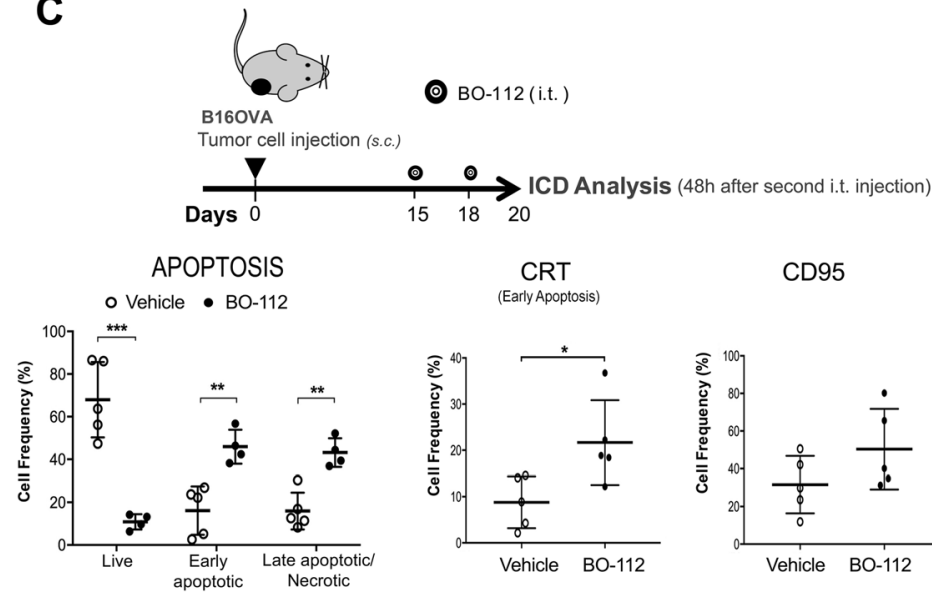

CRT

(Early Apoptosis)

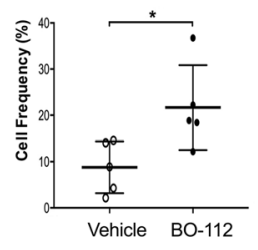

CD95

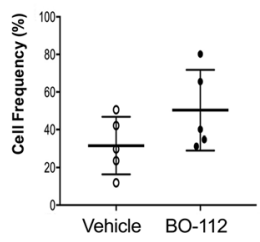

MHC-I

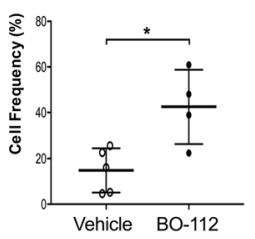

Fig. 2 BO-112 induces immunogenic cell death. The characterization of tumor cell death (apoptosis, necrosis, immunogenic cell death) induced by BO-112 was investigated in vitro and in vivo. a. and $\mathbf{b}$. B16-OVA cells ( $10^{5}$ cells/well) were cultured alone or with BO- 112 or Poly IC (0.25, 0.5 and $1 \mu \mathrm{g} / \mathrm{ml})$, for 24 and $48 \mathrm{~h}$. a. Apoptosis and necrosis were analyzed by flow cytometry upon staining with Annexin $V$ and 7AAD. b.

Immunogenic cell death (ICD) hallmarks were analyzed by flow cytometry studying cell surface expression of MHC-I, CD95 and Calreticulin and by measuring HMGB1 release. c. B16-OVA tumor bearing mice were intratumorally treated with BO-112 or vehicle ( $n=5$ per group). The diagram shows the schedule of the experiment. Graphs show that intratumoral administration of BO-112 leads to a significant increase in tumor cell apoptosis and necrosis (left) and also promotes the expression of ICD-associated markers on tumor cells. ${ }^{*} P<0.05,{ }^{* *} P<0.01{ }^{* *} P<0.001$

\section{Intratumoral BO-112 increases CD8 ${ }^{+}$tumor infiltrating lymphocytes and $\mathrm{CD} 8 / \mathrm{Treg}$ ratios}

Intratumoral BO-112 therapeutic effects could be the result of direct tumor cell death or the result of enhanced antitumor immune responses or a combination of both factors. To start addressing this question, we studied the composition of the lymphoid infiltrates following BO-112 intratumoral delivery in B16-OVA-derived tumors (Fig. 3a and Additional file 6: Figure S5). Interestingly, we observed changes in the leukocyte tumor microenvironment 
A

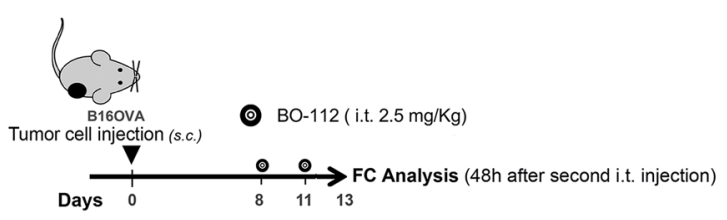

B
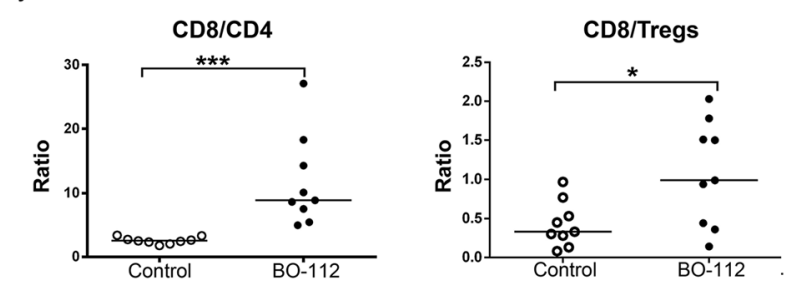

C CD8+ T CELLS \%CD8+/CD45+
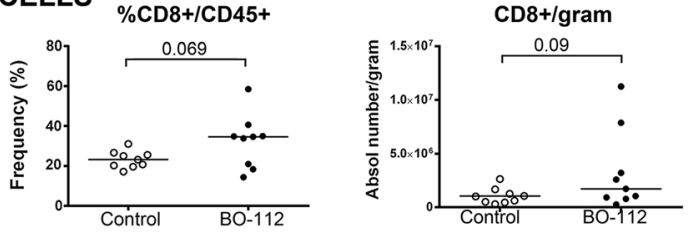

CD4+ T CELLS

$\% \mathrm{CD} 4+/ \mathrm{CD} 45+$

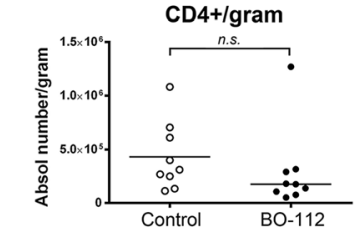

TREGS
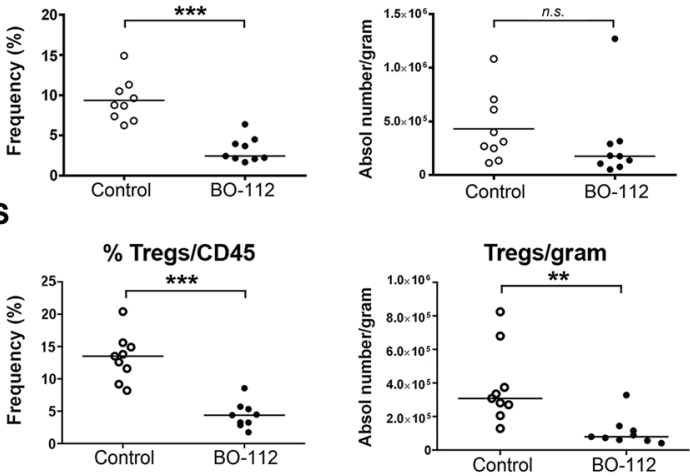

D CONTROL

BO-112

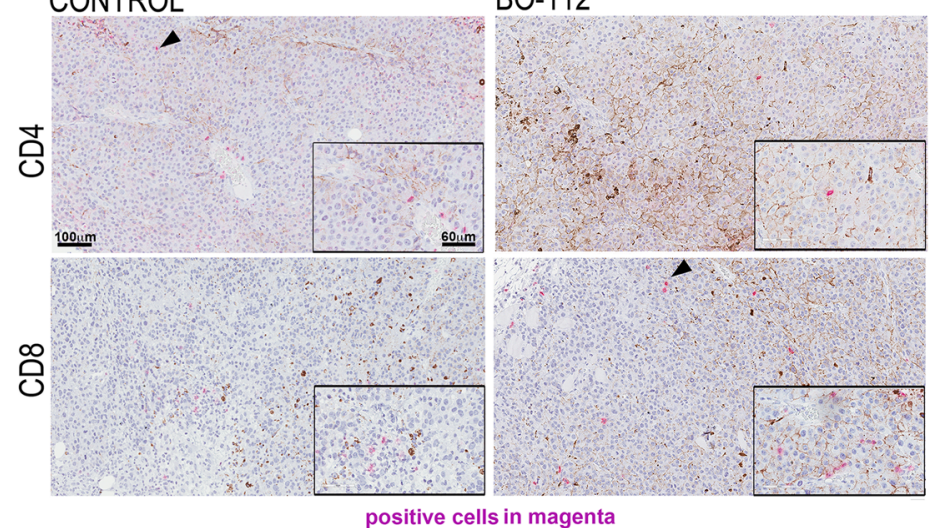

Fig. 3 BO-112 intratumoral injection enhances T lymphocyte infiltrates. a. Schematic representation of the experiments to surgically harvest tumors following treatment to generate cell suspensions that were analyzed by flow cytometry. $\mathbf{b}$. CD8/CD4 and CD8/Treg ratios in cell suspensions. c. Percentage of $\mathrm{CD}^{+}, \mathrm{CD}^{+}$and $\mathrm{CD}^{2} 5^{+} \mathrm{FOXP3}^{+}$over total intratumoral $\mathrm{CD} 45^{+}$leukocytes and absolute numbers per gram of tumor tissue. $\mathbf{d}$. Representative microphotographs of CD4 and CD8 immunohistochemistry analyses of sections derived from B16-OVA tumors treated as indicated. Scale bar of the main microphotograph: $100 \mu \mathrm{m}$. Scale bar of the inset: $60 \mu \mathrm{m}$. Positive cells are stained in magenta. ${ }^{*} P<0.05,{ }^{*} P<0.01 * * P<0.001$ 
in favor of $\mathrm{CD}^{+}$cells in proportion to $\mathrm{CD} 4^{+} \mathrm{T}$ cells and regulatory $\mathrm{T}$ cells (Tregs) (Fig. 3b). In fact, higher numbers of $\mathrm{CD}^{+}$cells could be observed per gram of tumor tissue, whereas $\mathrm{FOXP}^{-} \mathrm{CD}^{+}$and $\mathrm{FOXP}^{+} \mathrm{CD} 25^{+} \mathrm{CD} 4^{+}$Tregs cells decreased (Fig. 3c). These infiltrates were evenly distributed in the tumors as representative microphotographs in Fig. 3d show.

\section{Efficacy of intratumoral BO-112 given unilaterally to bilaterally tumor-bearing mice in conjunction with systemic anti-CD137 and anti-PD-L1 monoclonal antibodies}

The intratumoral route in all detectable tumors might be possible in oligometastatic patients, but impossible in most advanced cancer patients. Furthermore, microscopic metastatic disease will not be amenable to intratumoral treatment. Therefore, we performed experiments in B16-OVA tumor-bearing mice in which only one of the subcutaneously engrafted tumors was treated (Fig. 4a). These mice were intraperitoneally co-treated with control antibody or immunomodulatory mAbs agonistic for CD137 [43] or antagonistic for PD-L1 [44]. As seen in Fig. 4b and c, BO-112 exerted clear local control of the disease as compared to vehicle. Such local control was enhanced to some extent by both anti-CD137 and anti-PD-L1 mAbs, which did not show any meaningful therapeutic activity by themselves, as shown in their combination with intratumoral control vehicle.

When examining distant tumors (non-injected with BO-112), some degree of tumor-growth control by BO-112 was observed with death of mice being postponed for approximately 1-2 weeks (Fig. $4 \mathrm{~b}$ and c). Furthermore, when systemic anti-CD137 mAb was used such distant tumor control was further enhanced, although not significantly in the case of anti-PD-L1-treated mice. In this difficult-to-treat melanoma model, our data argue in favor of systemic antitumor activity that might be potentiated by combinations with other immuno-oncology agents.

In B16-OVA tumors intratumorally treated with BO-112, we were able to observe increases in the expression levels of the targets for immunomodulatory mAbs on tumor-infiltrating $\mathrm{T}$ cells $48 \mathrm{~h}$ following the second BO-112 administration. PD-1 expression markedly rose on $\mathrm{CD}^{+} \mathrm{T}$ cells, while CD137 expression was also increased, albeit to a lesser extent (Additional file 7: Figure S6A). Curiously, CD137 was clearly upregulated on NK lymphocytes retrieved from treated tumors. Moreover, PD-L1 levels of surface expression increased on $\mathrm{T}$ cells $\left(\mathrm{CD}^{+}\right.$and $\left.\mathrm{CD} 4^{+}\right)$, as well as on NK cells (Additional file 7: Figure S6A). Therefore BO-112 intratumoral treatment at least locally upregulates the targets for the mAbs used in the immunotherapy combinations, including $\mathrm{CD}^{+}{ }^{+}$cells that co-expressed both PD-1 and CD137 on their surface (Additional file 7: Figure S6B).
In MC38-derived tumors, BO-112 intratumor injections clearly induced an enrichment of $\mathrm{PD} 1^{+}, \mathrm{CD} 137^{+}$and double positive $\mathrm{CD} 137^{+} \mathrm{PD}-1^{+}$among $\mathrm{CD} 8+\mathrm{T}$ cells, although total CD8+ were not augmented (Additional file 8: Figure S7A and $\mathrm{B}$ ). In line with these findings, in B16-OVA-derived tumors, FOXP3 ${ }^{-} \mathrm{CD} 4^{+}$and $\mathrm{FOXP} 3{ }^{+} \mathrm{CD} 25^{+} \mathrm{CD} 4^{+}$cells per gram of tumor tissue were reduced following $\mathrm{BO}-112$ treatment (Additional file 8: Figure S7C).

\section{Intratumoral BO-112 enlarges tumor-draining lymph nodes containing abundant $\mathrm{CD}^{+} \mathrm{T}$ cells}

Intratumoral release not only reaches the tumor microenvironment itself, but also drains to lymph nodes. In our hands, two intratumoral injections of BO-112 at therapeutic doses (Fig. 5a) in B16-OVA-tumor bearing mice resulted in prominent draining lymph node enlargements (Fig. 5b), as a result of an enhanced contents of $\mathrm{CD} 45^{+}$ leukocytes. Again, $\mathrm{CD}^{+} / \mathrm{CD}^{+}$and $\mathrm{CD} 8^{+} /$Treg ratios were markedly increased by treatment in most tumors, while non-draining lymph nodes remained normal (data not shown). Of interest, while numbers of effector $\mathrm{CD}^{+}$ and $\mathrm{CD} 4^{+} \mathrm{T}$ cells rose, Tregs remained stable (Fig. $5 \mathrm{c}$ ).

\section{Antitumor activity of intratumoral $\mathrm{BO}-112$ requires IFNy} and correlates with increases in tumor-reactive $\mathrm{CD} 8^{+} \mathrm{T}$ cells Results from tumor infiltrates suggested that an important component in the therapy was mediated by the immune system. To determine which cell subsets were involved in the BO-112 tumor growth delay, depletion experiments were performed in MC38 and B16-OVA tumors. Antitumor effects on MC38-derived tumors completely disappeared when $\mathrm{CD}_{\beta}{ }^{+} \mathrm{T}$ cells were depleted in vivo, while $\mathrm{CD} 4+\mathrm{T}$ cells were dispensable (Additional file 9: Figure S8A and B). By contrast, single CD8 subset depletion induced only a partial loss of efficacy and single CD4+ and NK+ subset depletion had not impact in BO-112-mediated antitumor response in B16-OVA mouse models. (Additional file 10: Figure S9 A and B). Interestingly, triple depletion of NK1.1+, CD4+ and CD8+ had a major impact on BO-112 therapeutic effects (Additional file 10: Figure S9 B), although such loss of efficacy was not complete, indicating that other mechanisms are involved in $\mathrm{BO}-112$-induced antitumor response. In addition, myeloid $\mathrm{Gr}-1+$ cells were dispensable (Additional file 10: Figure S11 A and B). Depletions were verified in peripheral blood by flow cytometry (Additional file 9: Figure S8C and Additional file 10: Figure S9 $\mathrm{C}$ and $\mathrm{D}$ ), and $\mathrm{Gr}-1$ depletion was additionally verified in both blood and tumor (Additional file 11: Figure S10 C and D).

The immunotherapeutic effects mediated by $\mathrm{CD}^{+} \mathrm{T}$ cells are usually dependent on IFN $\gamma$ and the critical contribution of this cytokine to intratumoral BO-112 efficacy was revealed in BO-112-treated B16-F10 tumors 
A
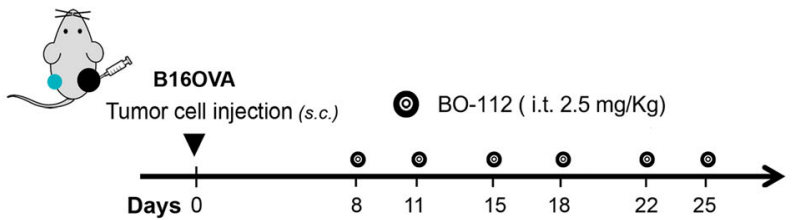

B
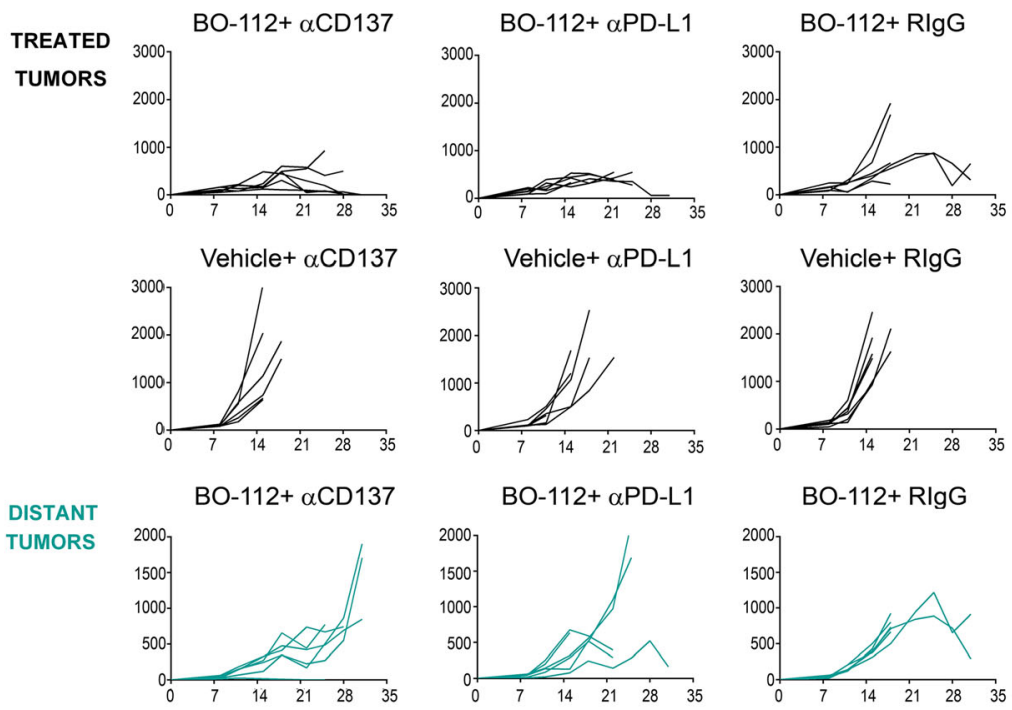

BO-112+ RIgG
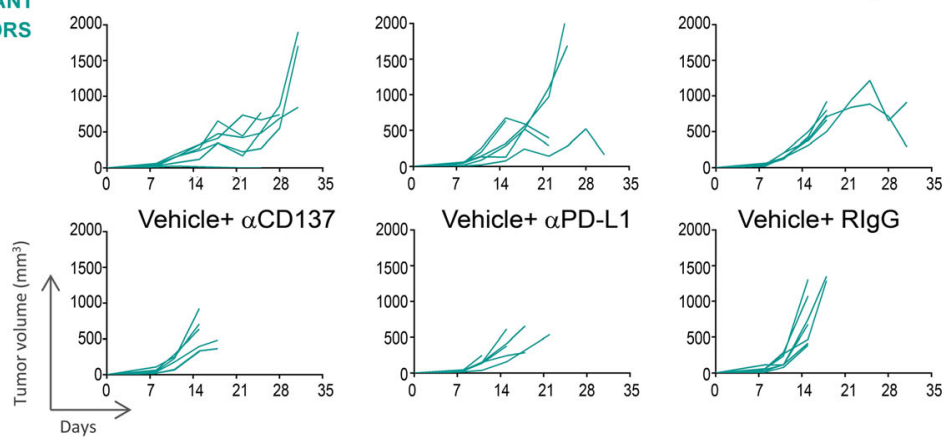

C

TREATED TUMORS
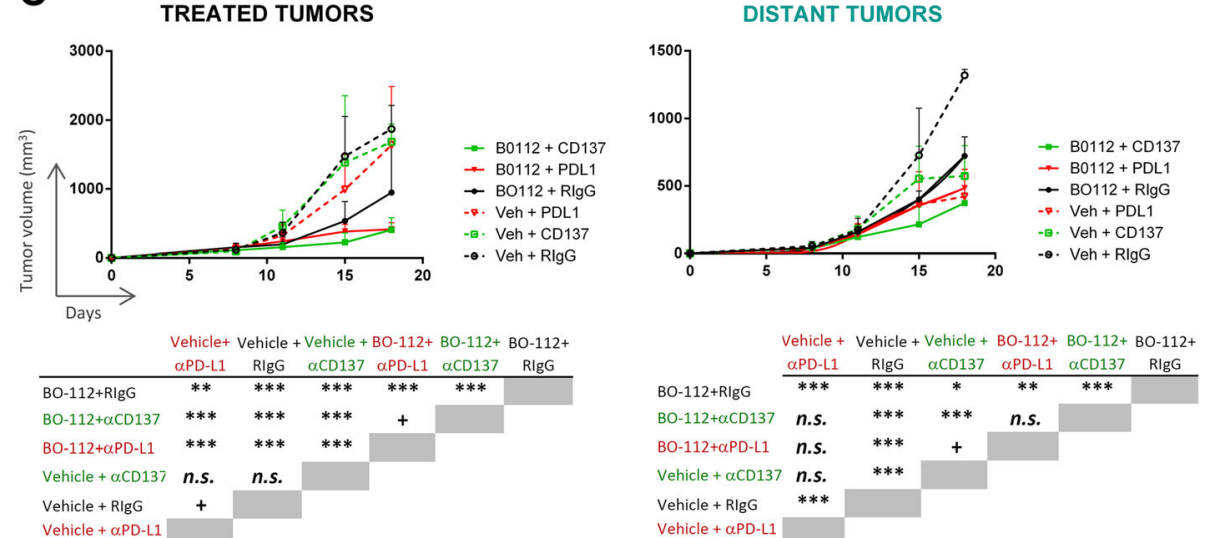

Fig. 4 Immunotherapeutic effects of combinations of intratumoral BO-112 with systemic anti-CD137 or anti-PD-L1 monoclonal antibodies. a. Schematic representation of experiments in mice bearing two B16-OVA-derived tumors engrafted on opposite flanks and intratumorally treated with BO-112 only in the right lesion and with intraperitoneal administrations of immunomodulatory monoclonal antibodies as indicated. $\mathbf{b}$. Tumor volume follow-up of the injected and distant tumors in the different groups of treatment. c. Mean \pm SD summary indicating statistical significance of the listed comparisons. ${ }^{*} P<0.05,{ }^{*} P<0.01{ }^{* *} P<0.001$

when IFNY was systemically neutralized with a specific mAb (Additional file 11: Figure S10E).

Various tumor specificities of $\mathrm{CD}^{+} \mathrm{T}$ lymphocytes in TDLN and in the tumor microenvironment can be monitored with $\mathrm{H}-2 \mathrm{k}^{\mathrm{b}}$ tetramers refolded with wellstudied immunodominant CTL epitopes such as Ovalbumin (OVA) and TRP2 in B16-OVA, and gp70 in the MC38 model. In this regard, injections of BO-112 into 
A
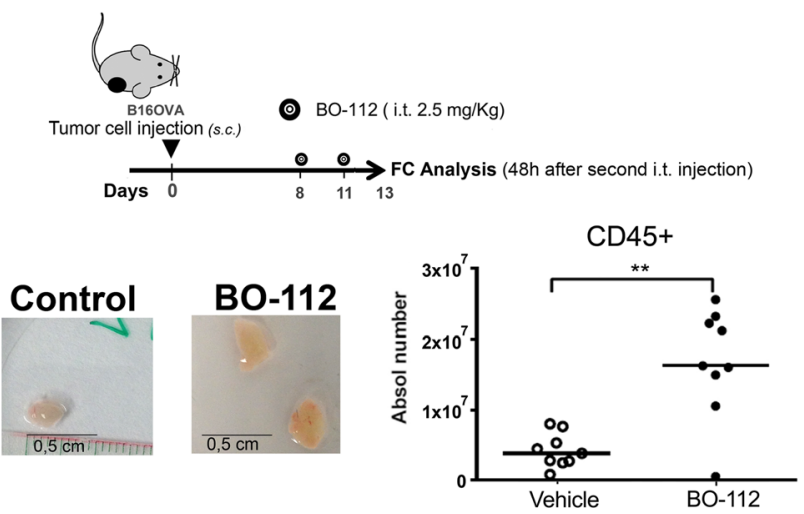

B

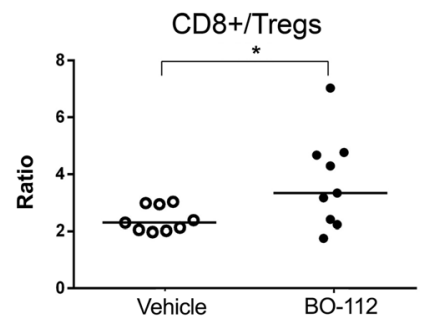

C
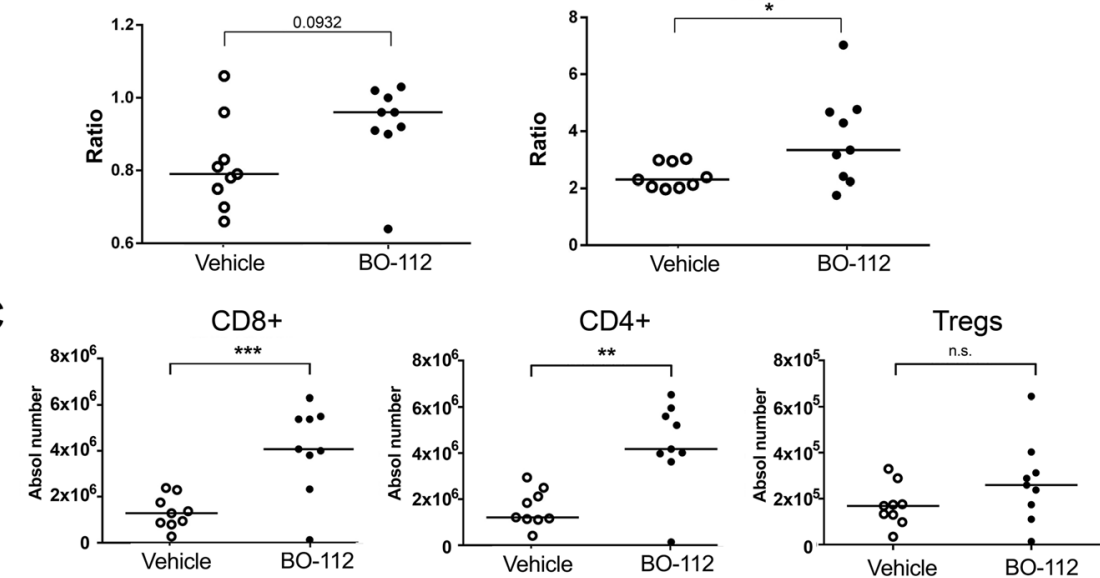

D

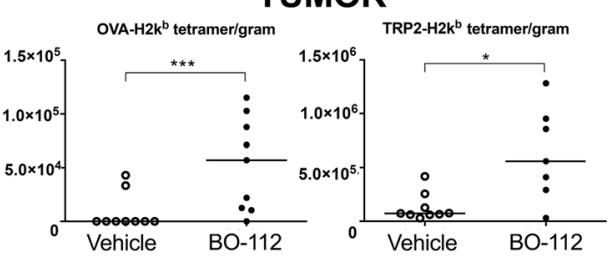

E

TDLN

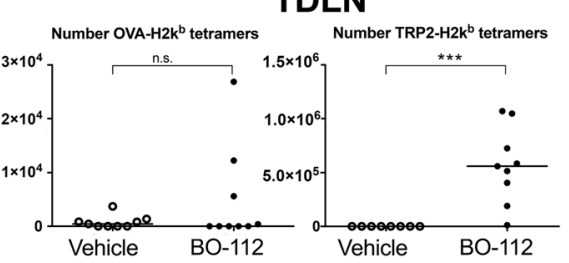

Fig. 5 BO-112 intratumoral injection induces tumor-draining lymph node enlargement and increases CD8 T cells recognizing specific antigens. a. Scheme of experimental treatment showing representative size of TDLN and their total leukocyte content in the graph comparing mice treated intratumorally with BO-112 or control vehicle. $\mathbf{b}$ and $\mathbf{c}$.: Analysis by flow cytometry of individual TDLN cell suspensions. $\mathbf{b}$. CD8 to CD4 ratios and CD8/Treg ratios. $\mathbf{c}$. represents the absolute number of the indicated T-cell subsets in TDLNs. $\mathbf{d}$. Class I MHC tetramer stainings to identify T cells recognizing OVA-specific epitope and TRP-2 among CD8 T cells per gram of malignant tissue in mice bearing B16-OVA tumors. e Class I MHC tetramer stainings to identify the numbers OVA- and TRP2-specific CD8+ T cells in TDLN. Absolute numbers are provided for antigen-specific CD8 T cells. ${ }^{*} P<0.05,{ }^{* *} P<0.01{ }^{* *} P<0.001$

B16-OVA tumors resulted in increased contents $\mathrm{CD} 8^{+} \mathrm{T}$ cells recognizing TRP-2 and OVA as a surrogate tumor antigen in tumors (Fig. 5d) and in TDLN (Fig. 5e).

In line with these findings in B16-OVA models, a remarkable increase of gp70-specific intratumor CD8+ $\mathrm{T}$ cells was found in MC38-tumor bearing mice treated intratumorally with BO-112 (Additional file 12 Figure 11).
Intratumoral BO-112 induces an IFNa/ $\beta$-related transcriptomic profile and type I interferon as well as cDC1 dendritic cells are required for antitumor effects Previous reports have linked Poly I:C delivery to IFN $\alpha / \beta$ release $[45,46]$. We genome-wide analyzed the mRNAs expressed in B16-OVA tumors $48 \mathrm{~h}$ following the second intratumoral BO-112 administration in comparison with control Vehicle (Fig. 6a and Additional file 13: Figure S12) 
A

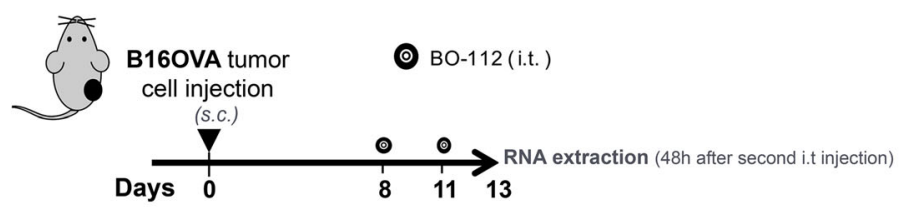

B

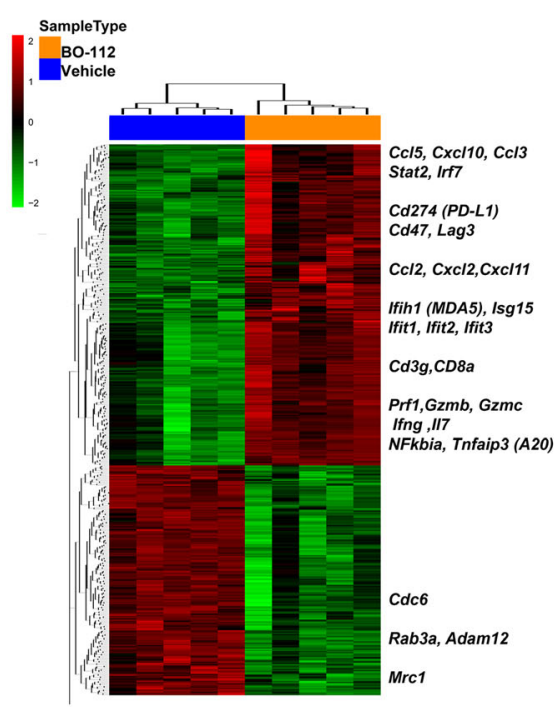

C

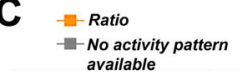

Activation of IRF by cytosolic PRRs Interferon signaling

Retinoic adcid mediated apoptosis signaling Communication between Innate and
Adaptive Immmune cells ox40 Signallind Pathway Role of PRRs in recognition of Death receptor signaling

Granulocyte adhesion and P38 MAPK signaling

Acute phase response signalling

Antigen presentation pathway

LXR/RXR Activation

Toll-like receptor signaling

Granzyme A signaling

Role of Rig--like receptors in antivir innate immunity
Th1 Pathway

Crosstalk between dendritic cells
and NKs

IL-6 signaling

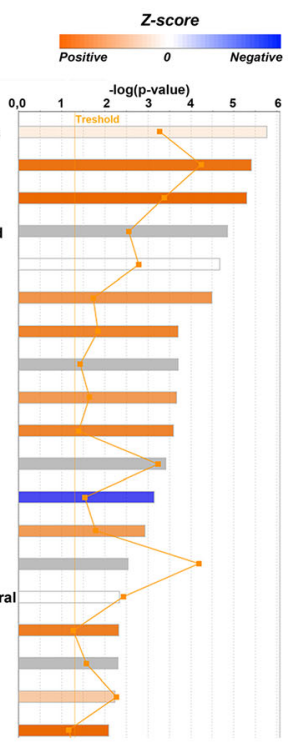

D

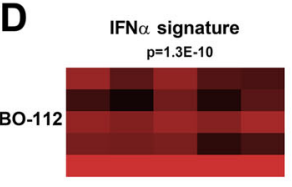

Vehicle

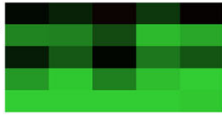

Ifit1 Ifit2 Iff7 Mx1 Tap2
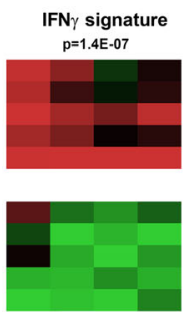

Cxcl10 Cxcl11 lfng Prf

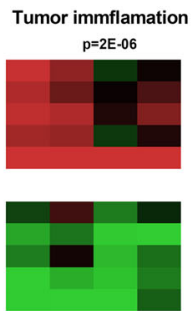

Cc15 Cd274 Cd8a Lag3
TILS

infiltration/activation T-cell effector genes

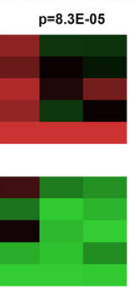

Cd274 Cd8a Ifng

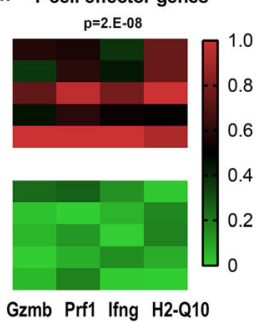

Fig. 6 Intratumoral BO-112 induces potent type-I IFN-related transcriptomic changes. a. Mice bearing B16-OVA tumors were treated with intratumoral BO-112 or vehicle ( $n=5$ per group) and total RNA was extracted as indicated to be genome-wide analyzed by gene expression microarrays. Differentially expressed transcripts were obtained by Linear Models for Microarray Data (LIMMA) analysis (b). Hierarchical clustering of differentially expressed genes between both experimental conditions. Most relevant genes for immune functions are indicated as upregulated by BO-112. c. Top canonical pathways upregulated by BO-112 treatment as defined by Ingenuity Pathway Analysis of the differentially expressed transcripts. d. Heat map representing enrichment analyses of key previously described signatures for IFNa and IFNץ stimulation, for tumor cell infiltration and activation of TILs as well as T-cell effector-related transcripts

and in B16-OVA cell cultures following $24 \mathrm{~h}$ incubation with BO-112 of Vehicle (Additional file 14: Figure S13).

As expected, a very clear differential gene expression profile was found upon intratumoral BO-112 administration, involving key immune-response genes whose expression rose in a clear-cut pattern, as shown in the hierarchical clustering of Fig. $6 \mathrm{~b}$ and extended data in Additional file 15: Table S2. The differentially expressed gene set was significantly enriched in immune-response gene signatures involved in Interferon signaling and retinoic acid-mediated apoptosis (Fig. 7c and Additional file 16:
Table S3). The BO-112-induced transcriptional profile was also enriched in previously reported gene signatures that suggest infiltration by activated immune cells and cytolytic activity [38, 39] (Fig. 6d). This gene expression pattern is comparable to that induced by poly I:C, since it best fits in Ingenuity pathway analysis (IPA) what has been described for cell exposure to Poly I:C, as expected from stimulation of TLR3 and cytosolic pattern recognition receptors (PRRs) (Additional file 17: Table S4).

Key genes involved in IRF activation by cytosolic PRRs, IFN signaling, retinoic acid-mediated apoptosis were among 


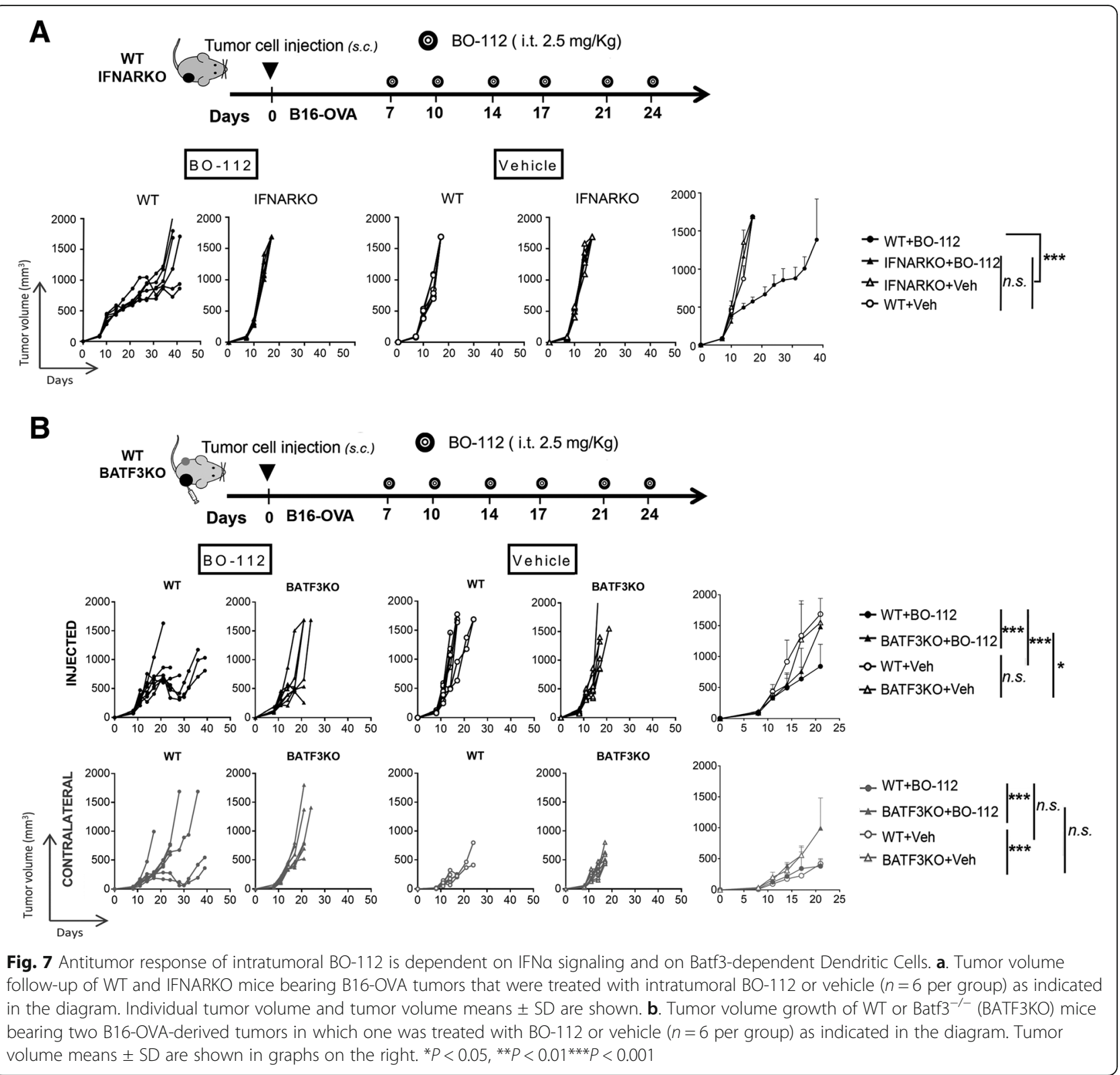

the 254 differentially expressed genes shared between in vitro and in vivo experiments (Additional file 14: Figure S13), while other key immunoregulatory genes were upregulated by BO-112 in B16-OVA cell cultures (Additional file 14: Figure S13B and Additional file 18: Table S5).

All these effects on the transcriptomic profiles speak of an excellent mimicry of viral infection in the BO-112-injected tumor microenvironment conducive to the enhancement of anti-tumor cytotoxic T-cell responses.

As suggested by the observed induced expression of IFN-I, IFNARKO mice bearing B16-OVA tumors were found not to respond to intratumor BO-112, thereby providing evidence for the key role of IFN $\alpha / \beta$ signaling for the antitumor response induced by BO-112 (Fig. 7a). Tumor antigen crosspriming is known to be up-regulated by type I IFN and TLR3 function. CD8 T-cell crosspriming is critically mediated by so called conventional type-I dendritic cells (cDC1) that are absent in $\mathrm{BATF}^{-/-}$mice [33]. Experiments of intratumoral treatment with BO-112 performed in BATF-3 $3^{-/-}$mice bearing two B16-OVA tumors (in which one was left uninjected) showed that this cDC-1 subset is crucial for the therapeutic response to local BO-112, since the progression delay mediated by in situ delivery of BO-112 was completely lost when compared to wild type mice (Fig. 7b). 


\section{Discussion}

In this study we have explored BO-112, a nanoparticled form of Poly I:C administered via the intratumoral route. Intratumoral delivery of immunotherapy came to age with the FDA approval of the HSV-1 vector T-vec for locally advanced or metastatic melanoma [47]. However viral vectors are immunogenic and often encode pathogenic factors that downregulate cellular immunity. Viral-like nanoparticles such as BO-112 have the theoretical advantage of lacking immunogenicity, thus theoretically permitting limitless dose repetition. Moreover, they do not need the logistical and safety cautions that must be taken into account with viruses or modified recombinant viruses. In addition, a very strong dsRNA immunostimulatory profile proceeds unchecked by any interference from counteracting viral immunosuppressive proteins.

In our hands, BO-112 is therapeutically active when used intratumorally, showing anti-tumoral efficacy in experimental settings in which intratumoral Poly I:C did not. This offers advantages since doses can be kept far from toxic thresholds, while achieving at least local antitumor effects. Interestingly, similar subcutaneous dosing of BO-112 shows no efficacy as compared to intratumoral release. Consistent with other forms of Poly I:C, intratumoral BO-112 is safe in mice and achieves marked tumor control of injected transplanted tumors including poorly immunogenic variants.

Mechanistic studies dissecting the mode of action result in the following model: first, BO-112 induces cell death in a small fraction of tumor cells in the context of alarmins denoting stressful non-programmed cell death [42]. This may result in tumor antigen release and crosspresentation by professional antigen-presenting cells [48] including BATF-3 dependent $\mathrm{c}-\mathrm{DC} 1$. At the same time, strong IFN $\alpha / \beta$ release and other proinflammatory mediators act as a local adjuvant in this context of in-situ vaccination $[11,49]$. As a consequence, a tumor-specific CD8 immune response is mounted or augmented to the point of controlling tumor progression, both in the locally injected lesion and to some extent in distantly implanted tumor nodules. This is consistent with increases of tumor-specific CD8 T cells in the tumor microenvironment and TDLNs.

Mechanisms aside adaptive immunity are operational in the treatment as seen upon simultaneous depletion of $\mathrm{T}$ and NK lymphocytes. On the one hand there are direct cytotoxic effects to tumor cells and on the other there might be effects on the functionality of innate immune cells other than NK lymphocytes.

Other TLR [1] and STING [50] agonists are being developed for intratumoral injection in the clinic (for instance, the following ongoing clinical trials registered in clinicaltrial.gov: NCT02927964; NCT02423863; NCT02501473; NCT03172936). However, the induction of immunogenic cell death to a certain level could be an important advantage in the case of BO-112. It remains to be seen which, or which combination, of agonists to PRRs behaves as the most beneficial when injected intratumorally.

In the era of checkpoint inhibitors, combinations of local agents and systemic immunomodulatory mAbs make much sense [51]. In the case of intratumoral BO-112, we observe some additive effects with anti-PD-L1 and anti-CD137 mAbs, that were not truly synergistic. In this regard, clinical reports on results of the anti-PD-1 mAb combinations with other TLR agonists such as G100, CpG oligonucleotides and STING agonists given locally are eagerly expected. This is also the case of intratumoral oncolytic virus T-vec that in combination with pembrolizumab has shown remarkable responses in metastatic melanoma patients [52], being pending of the results of the randomized clinical trial MASTERKEY 265 in combination with pembrolizumab versus pembrolizumab alone (NCT02263508). In the case of BO-112, the expression of CD137 and PD-1/PD-L1 was increased on tumor infiltrating $\mathrm{T}$ and NK cells, a fact that hinted at the potential combinability of the dual local and systemic approach.

All things considered, we have observed that intratumoral BO-112 is active in local cancer immunotherapy. It remains to be studied what would be the best combination regimen, but for the time being $\mathrm{BO}-112$ is combined in the clinic with anti-PD-1 mAbs, since our results have been conductive to an ongoing clinical trial (NCT02828098) which is testing the safety and clinical activity of intratumoral BO-112 either as a single agent or in combination with nivolumab or pembrolizumab checkpoint inhibitors. The transcriptomic gene-expression profile induced by BO-112 in engrafted mouse tumors offers potential for pharmacodynamics biomarkers and, as a consequence, RNA expression assessments are being carried out in pre- and post-treatment biopsies of injected tumors taken from patients on trial.

\section{Conclusions}

Nanoplexed Poly I:C (BO-112) when locally injected induces immunogenic cell death in a fraction of tumor cells and exerts potent antitumor activity via strong induction of type I interferon and CD8 T-cell infiltrates in the tumor microenvironment. As a result of these findings intratumoral BO-112 is undergoing phase I/II clinical trials.

\section{Additional files}

Additional file 1: Table S1. Flow cytometry mAbs and other staining dyes employed for multiparametric flow cytometry analyses. (XLSX $12 \mathrm{~kb}$ )

Additional file 2: Figure S1. A representative BO-112 intensity size distribution is presented, that was determined by Dynamic Light Scattering $(\mathrm{DLS})$, a non-invasive technique for measuring the size of particles in suspension. 
Above of the DLS graph there is a cartoon representing the postulated structure of a BO-112 nanocomplex. (TIF $513 \mathrm{~kb}$ )

Additional file 3: Figure S2. BO-112 cytotoxic effects on human tumor cell lines. A. Cell viability experiments as in Fig. 1a showing the effects of various doses of BO-112 or Poly I:C on human tumor cell lines representing melanoma, colon cancer and breast cancer. B. Cell viability of B16-OVA, MC38, HCT 116 and HT-29 tumor cell lines upon incubation with increasing amounts of BO-112 for 24 and $48 \mathrm{~h}$. Cell viability was assessed by MTS assay. $\%$ Viability is referred to untreated cells. C. Early and late apoptosis assessment induced by BO-112 in two representative human tumor cell lines measured by flow cytometry as in Fig. 2a. (TIF $1168 \mathrm{~kb}$ )

Additional file 4: Figure S3. Intratumor delivery of polyethylenimine is unable to induce therapeutic effects. A. xCELLigence experiments as in Fig. 1a showing B16-OVA cell viability upon in vitro incubation with BO-112 or PEl in equivalent amounts as present in each BO-112 dose. B. Timeline showing the treatment schedule of intratumoral administrations of BO-112 or PEl in B16OVA models. Mice were injected subcutaneously with B16-OVA at day 0 $\left(5 \times 10^{5}\right.$ cells) in the right flank. When tumor size reached $80-100 \mathrm{~mm}^{3}$, animals were treated with PEl or BO-112 by injection into the tumor nodules (i.t). Plots show individual volume (length $\mathrm{x}$ width $/ 2$ ) for control (vehicle) and PEl and BO-112 treated mice. ${ }^{* *} P<0.001$. (TIF $4613 \mathrm{~kb}$ )

Additional file 5: Figure S4. BO-112 therapeutic effects require intratumoral administration. A. Timeline showing the schedule of experimental treatment. All mice were injected subcutaneously with B16OVA or MC38 murine colon carcinoma cells at day $0\left(5 \times 10^{5}\right.$ cells $)$ in the right flank. After mice randomization (when tumor size reached 80-100 $\mathrm{mm}^{3}$ ), animals were treated with BO-112 by injection into the tumor nodules (i.t) or by subcutaneous injection in the left flank (s.c). Plots show individual volume (length $\mathrm{x}$ width ${ }^{2} / 2$ ) for control (vehicle) and BO-112 treated mice, following i.t and s.c routes of drug administration as indicated. ${ }^{* * *} P<0.001$ (TIF $5096 \mathrm{~kb}$ )

Additional file 6: Figure S5. Gating strategy for flow cytometry analyses to study tumor infiltration in in vivo experiments. Flow cytometry plots of a representative sample showing the gating strategy for TIL analysis. (TIF $1065 \mathrm{~kb}$ )

Additional file 7: Figure S6. Expression of CD137, PD-1 and PD-L1 on infiltrating lymphocytes from BO-112-treated B16-OVA tumors. A. Flow cytometry analysis of the intensity of expression of surface PD-1, CD137 and PD-L1 on gated $\mathrm{CD}^{+}, \mathrm{CD}^{+}$and NK lymphocytes comparing tumors treated with $\mathrm{BO}-112$ or vehicle. B. Percentage of $\mathrm{CD} 8^{+}$cells coexpressing PD-1 and CD137 and their density per gram of tumor as analyzed following intratumoral treatment with $\mathrm{BO}-112$ or control vehicle. ${ }^{* *} P<0.01{ }^{* * *} P<0.001$ (TIF $1114 \mathrm{~kb}$ )

Additional file 8: Figure S7. BO-112 intratumoral injection enhances T lymphocyte infiltrates of MC38 tumors. A. Schematic representation of the experiments to generate cell suspensions of MC38 tumors that were analyzed by flow cytometry. B. Flow cytometry frequencies of PD-1 ${ }^{+}$, $\mathrm{CD} 137^{+}$and $\mathrm{PD}-1^{+} \mathrm{CD} 137^{+}$double positive CD8+ in the CD8+ T cell population infiltrating MC38 tumors after two intratumoral injections of BO-112, and absolute numbers of CD8 ${ }^{+} T$ cells per gram of tumor. $C$. Absolute number per gram of CD4 and CD25 + CD4+ Tregs per gram of tumor. ${ }^{*} P<0.05 * * P<0.01$ (TIF $3622 \mathrm{~kb}$ )

Additional file 9: Figure S8. CD8 depletion abrogate therapeutic effects of BO-112 intratumoral delivery in MC38-tumor bearing mice. A. Schematic representation of experiments on mice bearing MC38-derived tumors for lymphocyte subset depletion. B. Individual follow-up upon treatment with BO-112 or vehicle in mice depleted of CD8 or CD4 T cells by specific monoclonal antibodies. C. CD4+ and CD8+ depletion validation in peripheral blood of a representative group of animals was analyzed by flow cytometry during the experimental procedure. ${ }^{* *} P<0.01^{* * *} P<0.001$. (TIF $1913 \mathrm{~kb}$ )

Additional file 10: Figure S9. CD8, CD4 and NK depletion in B16-OVA tumor-bearing mice treated with intratumoral BO-112. A. Schematic representation of experiments on B16-OVA-tumor bearing mice that were depleted from the indicated lymphocyte subsets. B. Individual tumor volume follow-up in groups of mice intratumorally treated with with $\mathrm{BO}$ 112 or vehicle and depleted from CD8+, CD4+ or NK1.1+ cells separately or concomitantly. Lymphocyte cell subsets were selectively depleted by specific monoclonal antibodies. The corresponding statistical comparisons are summarized below the graphs. C. Representative dot plots of NK1.1+ CD4+ and CD8+ lymphocyte depletions as assessed in peripheral blood analyzed by flow cytometry during the experimental procedure. In D levels of depletion achieved in individual mice are shown. ${ }^{*} P<0.05{ }^{* *} P<$ $0.01{ }^{* *} P<0.001$. (TIF $2355 \mathrm{~kb}$ )

Additional file 11: Figure S10. Gr-1 depletion and IFNy neutralization in tumor-bearing mice treated with intratumoral BO-112. A. Schematic representation of experiments on B16-OVA-tumor bearing mice that were treated for $\mathrm{Gr}-1$ depletion. B. Tumor volume follow-up in mice depleted of $\mathrm{Gr}-1$ cells by a specific monoclonal antibody and intratumorally treated with with BO-112 or vehicle. C. Gr-1 depletion validation in B16-OVA tumors analyzed by flow cytometry on day 7 coinciding with the first intratumoral injection of BO-112. D. Gr-1+ depletion validation in peripheral blood performed as in C. E. Experiments in B16-F10 melanoma-bearing mice treated with intratumoral BO-112 or vehicle recording individual tumor sizes. When indicated, mice were given neutralizing anti-IFNy mAb or isotype control. ${ }^{* *} P<0.01{ }^{* * *} P<0.001$. (TIF $2920 \mathrm{~kb}$ )

Additional file 12: Figure S11. Increases of tumor-specific CD8+ T cells recognizing the gp70 tumor antigen following BO-112 injections into MC38 tumors. A. Diagram showing treatment schedule for MHC-I pentamer staining to identify gp70-specific CD8 T cells in mice bearing MC38 tumors. Frequencies of antigen-specific CD8 T cells infiltrating tumors (B.) and TDLNs (C.) are shown. ${ }^{*} P<0.05$. (TIF $2584 \mathrm{~kb}$ )

Additional file 13: Figure S12. Volcano Plot highlighting top differentially expressed genes (as per FC) in BO-112-treated B16-OVA tumors. RNA derived from B16-OVA tumors treated with intratumoral BO-112 or vehicle as indicated in Fig. 6 was analyzed by expression microarrays. Differentially expressed genes with a $|\log F C|>1$ and $p>0.01$ are considered differentially expressed in BO-112-treated B16-OVA tumors. (TIF $581 \mathrm{~kb}$ )

Additional file 14: Figure S13. Key immunoregulatory genes differentially expressed upon BO-112 intratumoral administration are also induced in B16-OVA cultures incubated with BO112. The B16-OVA cell line was incubated either with BO-112 or vehicle for $24 \mathrm{~h}$ and its RNA was genome wide analyzed with gene-expression microarrays. A. Venn diagram showing the differentially expressed genes that were shared with both in vitro and in vivo procedures (top) and top 19 canonical pathways in predicted by Ingenuity Pathway analysis (bottom). B. Hierarchical clustering of differentially expressed genes in B16-OVA after BO-112 incubation. (TIF $964 \mathrm{~kb}$ )

Additional file 15: Table S2. Differentially expressed genes obtained upon BO-112 intratumoral administration. Mice bearing B16-OVA tumors were treated with intratumoral BO-112 or vehicle as indicated in Fig. 6, and total RNA was extracted and analyzed by expression microarrays. Genes were selected as significant using a B-statistic cut-off $(B>0)$. (XLSX $195 \mathrm{~kb}$ )

Additional file 16: Table S3. Top canonical differentially regulated pathways induced by BO-112 intratumoral administration. Pathways from differentially expressed genes upon BO-112 intratumoral administration (selected as significant using a B-statistic cut-off B $>0$ ) were identified by Ingenuity Pathway Analysis. (XLS 35 kb)

Additional file 17: Table S4. Top 30 Upstream Regulators predicted to promote the differentially expression profile induced by BO-112 intratumoral administration. Upstream Regulators from differentially expressed genes upon BO-112 intratumoral administration (selected as significant using a B $>0$ cut-off) were identified by Ingenuity Pathway Analysis. (XLSX $17 \mathrm{~kb}$ )

Additional file 18: Table S5. Differentially expressed genes induced by BO-112 in B16-OVA in vitro. B16-OVA cell line was incubated either with BO-112 or vehicle for $24 \mathrm{~h}$ and its RNA was genome wide analyzed with gene-expression microarrays. Genes were selected as significant using a B $>0$ cut-off. (XLSX $241 \mathrm{~kb})$

\section{Abbreviations}

CDC1: conventional type-I dendritic cells; GEO: Gene Expression Omnibus; IFN-I: Type-I interferon; IFNy: Interferon-y; IPA: Ingenuity Pathway Analysis; LIMMA: Linear Models for Microarray Data; PRRs: cytosolic pattern recognition receptors; RMA: Robust Multichip Average algorithm; TDLN: Tumor-draining lymph nodes 


\section{Acknowledgements}

We thank Elisabeth Guruceaga and the Bioinformatics Facility of CIMA for the bioinformatics analysis and for technical assistance related with bioinformatic analysis. Eneko Elizalde is acknowledged for excellent animal facility work. We are grateful for scientific discussions and critical reading from Dr. Pedro Berraondo, Dr. Ana Rouzaut and Dr. David Sancho.

\section{Funding}

This study was financially supported by grants from MINECO (SAF2014-52361-R, FEDER/MICIU-AEI/SAF2017-83267-C2-1-R) to IM and JL-G. IM was also funded by European Commission VII Framework and Horizon 2020 programs (IACT FP7/ 2007-2013 grant agreement 602262 and PROCROP grant agreement 635122 respectively), Fundación de la Asociación Española Contra el Cáncer (AECC), Fundación BBVA. Bioncotech received funding from CDTI (IDI-20170635) to support this project.

\section{Availability of data and materials}

Microarray expression data can be downloaded from Gene Expression Omnibus (GEO) under the Series accession number GSE116078. Data generated or analyzed during this study are included in this published article (and its additional files).

\section{Authors' contributions}

$M P O, P L, I E, E B$ performed the in vitro experiments. MPO, PLC, SG, GP, IR and $\mathrm{CM}$ performed the in vivo and flow cytometry experiments. MPO, CM, SG and PL-C acquired the data. LP and MAA analyzed and interpreted the data. LP, MAA supervised the biological studies. MAA performed the bioinformatics pathway analyses. LP, IMR, MAA, MQ and IM designed the studies. LP, PL, MAA and IM drafted the work. IMR, MERR, JLP, LP, MQ and AT revised the manuscript IM and MQ supervised the entire study. All authors read and approved the final manuscript.

\section{Ethics approval and consent to participate}

Not applicable

\section{Consent for publication}

Not applicable

\section{Competing interests}

$M Q, L P, P L C$ and MPO are full time employees in Bioncotech. IM reports receiving commercial research grants from BMS, Alligator and Roche and serves as a consultant/advisory board member for BMS, Merck-Serono, Roche-Genentech, Genmab, Incyte, Bioncotech, Tusk, Numab, Genmab, Molecular partners, F-STAR, Alligator, Bayer and AstraZeneca.

\section{Publisher's Note}

Springer Nature remains neutral with regard to jurisdictional claims in published maps and institutional affiliations.

\footnotetext{
Author details

${ }^{1}$ Center for Applied Medical Research (CIMA), University of Navarra, Avenida Pio XII, 55, 31008 Pamplona, Spain. ${ }^{2}$ Bioncotech Therapeutics S.L, Valencia, Spain. ${ }^{3}$ Medical Oncology Department, Hospital General Universitario Gregorio Marañón, Madrid, Spain. ${ }^{4}$ Clínica Universidad de Navarra, Pamplona, Spain. ${ }^{5}$ CIBERONC, Madrid, Spain. ${ }^{6}$ IDISNA, Instituto de investigación de Navarra, Pamplona, Spain.
}

Received: 7 July 2018 Accepted: 15 March 2019

Published online: 02 May 2019

\section{References}

1. Aznar MA, Tinari N, Rullan AJ, Sanchez-Paulete AR, Rodriguez-Ruiz ME, Melero I. Intratumoral delivery of immunotherapy-act locally, Think Globally. J Immunol. 2017:198(1):31-9.

2. Kaminski JM, Shinohara E, Summers JB, Niermann KJ, Morimoto A, Brousal J. The controversial abscopal effect. Cancer Treat Rev. 2005;31(3):159-72.

3. Marabelle A, Kohrt H, Caux C, Levy R. Intratumoral immunization: a new paradigm for cancer therapy. Clin Cancer Res. 2014;20(7):1747-56.

4. Maas RA, Van Weering DH, Dullens HF, Den Otter W. Intratumoral low-dose interleukin-2 induces rejection of distant solid tumour. Cancer Immunol Immunother. 1991;33(6):389-94.
5. Mahvi DM, Henry MB, Albertini MR, Weber S, Meredith K, Schalch $H$, et al. Intratumoral injection of IL-12 plasmid DNA--results of a phase I/IB clinical trial. Cancer Gene Ther. 2007;14(8):717-23.

6. Ott PA, Hodi FS. Talimogene Laherparepvec for the treatment of advanced melanoma. Clin Cancer Res. 2016;22(13):3127-31.

7. Heo J, Reid T, Ruo L, Breitbach CJ, Rose S, Bloomston M, et al. Randomized dose-finding clinical trial of oncolytic immunotherapeutic vaccinia JX-594 in liver cancer. Nat Med. 2013;19(3):329-36.

8. Fransen MF, Ossendorp F, Arens R, Melief CJ. Local immunomodulation for cancer therapy: providing treatment where needed. Oncoimmunology. 2013;2(11):e26493.

9. Brody JD, Ai WZ, Czerwinski DK, Torchia JA, Levy M, Advani RH, et al. In situ vaccination with a TLR9 agonist induces systemic lymphoma regression: a phase I/II study. J Clin Oncol. 2010;28(28):4324-32.

10. Rodriguez-Ruiz ME, Perez-Gracia JL, Rodriguez I, Alfaro C, Onate C, Perez G, et al. Combined immunotherapy encompassing intratumoral poly-ICLC, dendritic-cell vaccination and radiotherapy in advanced cancer patients. Ann Oncol. 2018;29(5):1312-9.

11. Sagiv-Barfi l, et al. Eradication of spontaneous malignancy by local immunotherapy. Sci Transl Med. 2018;10(426). https://doi.org/10.1126/ scitransImed.aan4488

12. Kawai T, Akira $\mathrm{S}$. The role of pattern-recognition receptors in innate immunity: update on toll-like receptors. Nat Immunol. 2010;11(5):373-84.

13. Kawai T, Akira S. Toll-like receptor and RIG-l-like receptor signaling. Ann N Y Acad Sci. 2008;1143:1-20.

14. Riviere $Y$, Hovanessian $A$. Response of $L-1210$ tumor in mice toward treatment with interferon or poly(I) X poly(C). J Interf Res. 1983;3(4):417-24.

15. Hilleman MR. Prospects for the use of double-stranded ribonucleic acid (poly l:C) inducers in man. J Infect Dis. 1970;121(2):196-211.

16. Takemura R, Takaki H, Okada S, Shime H, Akazawa T, Oshiumi H, et al, Polyl:C-induced, TLR3/RIP3-dependent necroptosis backs up immune effector-mediated tumor elimination in vivo. Cancer Immunol Res. 2015; 3(8):902-14

17. Sanchez-Paulete AR, Cueto FJ, Martinez-Lopez M, Labiano S, MoralesKastresana A, Rodriguez-Ruiz ME, et al. Cancer immunotherapy with immunomodulatory anti-CD137 and anti-PD-1 monoclonal antibodies requires BATF3-dependent dendritic cells. Cancer Discov. 2016;6(1):71-9.

18. Amos SM, Pegram HJ, Westwood JA, John LB, Devaud C, Clarke CJ, et al. Adoptive immunotherapy combined with intratumoral TLR agonist delivery eradicates established melanoma in mice. Cancer Immunol Immunother. 2011;60(5):671-83.

19. Martins KA, Bavari S, Salazar AM. Vaccine adjuvant uses of poly-IC and derivatives. Expert Rev Vaccines. 2015;14(3):447-59.

20. Sabbatini P, Tsuji T, Ferran L, Ritter E, Sedrak C, Tuballes K, et al. Phase I trial of overlapping long peptides from a tumor self-antigen and poly-ICLC shows rapid induction of integrated immune response in ovarian cancer patients. Clin Cancer Res. 2012:18(23):6497-508.

21. Celis E. Toll-like receptor ligands energize peptide vaccines through multiple paths. Cancer Res. 2007:67(17):7945-7.

22. Ott PA, Hu Z, Keskin DB, Shukla SA, Sun J, Bozym DJ, et al. An immunogenic personal neoantigen vaccine for patients with melanoma. Nature. 2017; 547(7662):217-21

23. Armstrong JA, McMahon D, Huang XL, Pazin GJ, Gupta P, Rinaldo CR Jr, et al. A phase I study of ampligen in human immunodeficiency virus-infected subjects. J Infect Dis. 1992;166(4):717-22.

24. Salem ML, Kadima AN, Cole DJ, Gillanders WE. Defining the antigen-specific T-cell response to vaccination and poly(l:C)/TLR3 signaling: evidence of enhanced primary and memory CD8 T-cell responses and antitumor immunity. J Immunother. 2005:28(3):220-8.

25. Salem ML, El-Naggar SA, Kadima A, Gillanders WE, Cole DJ. The adjuvant effects of the toll-like receptor 3 ligand polyinosinic-cytidylic acid poly (l:C) on antigenspecific CD8+ T cell responses are partially dependent on NK cells with the induction of a beneficial cytokine milieu. Vaccine. 2006;24(24):5119-32.

26. Okada H, Butterfield LH, Hamilton RL, Hoji A, Sakaki M, Ahn BJ, et al. Induction of robust type-I CD8+ T-cell responses in WHO grade 2 low-grade glioma patients receiving peptide-based vaccines in combination with polyICLC. Clin Cancer Res. 2015;21(2):286-94.

27. Rodas IM, Ruiz MER, Cobo SL-T, Gracia JLP, Sarvise MP, Alvarez R, et al. LBA20Safety and immunobiological activity of intratumoral (IT) doublestranded RNA (dsRNA) BO-112 in solid malignancies: First in human clinical trial. Annals of Oncology. 2017;28(suppl_5):mdx440.013-mdx440.013. 
28. Caskey M, Lefebvre F, Filali-Mouhim A, Cameron MJ, Goulet JP, Haddad EK et al. Synthetic double-stranded RNA induces innate immune responses similar to a live viral vaccine in humans. J Exp Med. 2011;208(12):2357-66.

29. Salazar AM, Erlich RB, Mark A, Bhardwaj N, Herberman RB. Therapeutic in situ autovaccination against solid cancers with intratumoral poly-ICLC: case report, hypothesis, and clinical trial. Cancer Immunol Res. 2014;2(8):720-4.

30. Okada H, Kalinski P, Ueda R, Hoji A, Kohanbash G, Donegan TE, et al. Induction of CD8+ T-cell responses against novel glioma-associated antigen peptides and clinical activity by vaccinations with \{alpha\}-type 1 polarized dendritic cells and polyinosinic-polycytidylic acid stabilized by lysine and carboxymethylcellulose in patients with recurrent malignant glioma. J Clin Oncol. 2011;29(3):330-6.

31. Tormo D, Checinska A, Alonso-Curbelo D, Perez-Guijarro E, Canon E, Riveiro-

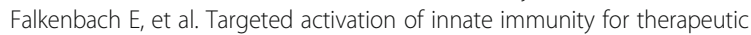
induction of autophagy and apoptosis in melanoma cells. Cancer Cell. 2009; 16(2):103-14.

32. Alonso-Curbelo D, Soengas MS. Self-killing of melanoma cells by cytosolic delivery of dsRNA: wiring innate immunity for a coordinated mobilization of endosomes, autophagosomes and the apoptotic machinery in tumor cells. Autophagy. 2010;6(1):148-50

33. Hildner K, Edelson BT, Purtha WE, Diamond M, Matsushita H, Kohyama M, et al. Batf3 deficiency reveals a critical role for CD8alpha+ dendritic cells in cytotoxic T cell immunity. Science. 2008;322(5904):1097-100.

34. Schilte C, Couderc T, Chretien F, Sourisseau M, Gangneux N, GuivelBenhassine F, et al. Type I IFN controls chikungunya virus via its action on nonhematopoietic cells. J Exp Med. 2010;207(2):429-42.

35. Irizarry RA, Bolstad BM, Collin F, Cope LM, Hobbs B, Speed TP. Summaries of Affymetrix GeneChip probe level data. Nucleic Acids Res. 2003;31(4):e15.

36. Ritchie ME, Phipson B, Wu D, Hu Y, Law CW, Shi W, et al. limma powers differential expression analyses for RNA-sequencing and microarray studies. Nucleic acids res. 2015;43(7):e47.

37. Gentleman RC, Carey VJ, Bates DM, Bolstad B, Dettling M, Dudoit S, et al. Bioconductor: open software development for computational biology and bioinformatics. Genome Biol. 2004;5(10):R80.

38. Ayers M, Lunceford J, Nebozhyn M, Murphy E, Loboda A, Kaufman DR, et al. IFN-gamma-related mRNA profile predicts clinical response to PD-1 blockade. J Clin Invest. 2017;127(8):2930-40.

39. Haymaker C, Uemura M, Hwu W, Murthy $R$, James M, Bhatta A, et al. TLR9 agonist harnesses innate immunity to drive tumor-infiltrating T-cell expansion in distant lesions in a phase 1/2 study of intratumoral IMO-2125 +ipilimumab in anti-PD1 refractory melanoma patients. (018). SITC 2017 Annual meeting November 8-12, 2017; National Harbor,MD, 2017.

40. Rodriguez-Ruiz ME, Rodriguez I, Garasa S, Barbes B, Solorzano JL, PerezGracia JL, et al. Abscopal effects of radiotherapy are enhanced by combined Immunostimulatory mAbs and are dependent on CD8 T cells and Crosspriming. Cancer Res. 2016;76(20):5994-6005

41. Garg AD, More S, Rufo N, Mece O, Sassano ML, Agostinis P, et al. Trial watch: immunogenic cell death induction by anticancer chemotherapeutics. Oncoimmunology. 2017;6(12):e1386829.

42. Galluzzi L, Buque A, Kepp O, Zitvogel L, Kroemer G. Immunogenic cell death in cancer and infectious disease. Nat Rev Immunol. 2017:17(2):97-111.

43. Chester C, Sanmamed MF, Wang J, Melero I. Immunotherapy targeting 41BB: mechanistic rationale, clinical results, and future strategies. Blood. 2018;131(1):49-57

44. Ribas A, Wolchok JD. Cancer immunotherapy using checkpoint blockade. Science. 2018;359(6382):1350-5.

45. Kelley KA, Pitha PM. Differential effect of poly rl.rC and Newcastle disease virus on the expression of interferon and cellular genes in mouse cells. Virology. 1985;147(2):382-93.

46. Matsumoto M, Seya T. TLR3: interferon induction by double-stranded RNA including poly(l:C). Adv Drug Deliv Rev. 2008;60(7):805-12.

47. Andtbacka RH, Kaufman HL, Collichio F, Amatruda T, Senzer N, Chesney J, et al. Talimogene Laherparepvec improves durable response rate in patients with advanced melanoma. J Clin Oncol. 2015;33(25):2780-8.

48. Sanchez-Paulete AR, Teijeira A, Cueto FJ, Garasa S, Perez-Gracia JL, SanchezArraez $A$, et al. Antigen cross-presentation and T-cell cross-priming in cancer immunology and immunotherapy. Ann Oncol. 2017;28(suppl_12):xii44-55.

49. Hammerich $L$, Bhardwaj $N$, Kohrt HE, Brody JD. In situ vaccination for the treatment of cancer. Immunotherapy. 2016;8(3):315-30.
50. Corrales L, Glickman LH, McWhirter SM, Kanne DB, Sivick KE, Katibah GE, et al. Direct activation of STING in the tumor microenvironment leads to potent and systemic tumor regression and immunity. Cell Rep. 2015;11(7): $1018-30$.

51. Melero I, Berman DM, Aznar MA, Korman AJ, Perez Gracia JL, Haanen J. Evolving synergistic combinations of targeted immunotherapies to combat cancer. Nat Rev Cancer. 2015;15(8):457-72.

52. Ribas A, Dummer R, Puzanov I, VanderWalde A, Andtbacka RHI, Michielin O, et al. Oncolytic Virotherapy promotes Intratumoral T cell infiltration and improves anti-PD-1 immunotherapy. Cell. 2017;170(6):1109-19 e10.

Ready to submit your research? Choose BMC and benefit from:

- fast, convenient online submission

- thorough peer review by experienced researchers in your field

- rapid publication on acceptance

- support for research data, including large and complex data types

- gold Open Access which fosters wider collaboration and increased citations

- maximum visibility for your research: over $100 \mathrm{M}$ website views per year

At $\mathrm{BMC}$, research is always in progress.

Learn more biomedcentral.com/submission 